\title{
Reduced-order modeling of fluid-structure interaction and vortex-induced vibration systems using an extension of Jourdain's principle
}

\author{
S. Mottaghi ${ }^{\mathrm{a}, \mathrm{b}, *}$ H. Benaroya ${ }^{\mathrm{a}, * *}$ \\ ${ }^{a}$ Department of Mechanical and Aerospace Engineering, Rutgers University, New \\ Brunswick, NJ, USA \\ ${ }^{b}$ Structures and Material Section, W. J. Hughes Technical Center, Federal Aviation \\ Administration, Atlantic City, NJ, USA
}

\begin{abstract}
A first-principles variational approach is proposed for reduced-order modeling of fluid-structure interaction (FSI) systems, specifically vortex-induced vibration (VIV). FSI has to be taken into account in the design and analysis of many engineering applications, yet a comprehensive theoretical development where analytical equations are derived from first principles is nonexistent. An approach where Jourdain's principle is modified and extended for FSI is used to derive reduced-order models from an extended variational formulation where assumptions are explicitly stated.
\end{abstract}

Two VIV models are considered: an elastically supported, inverted pendulum and a translating cylinder, both immersed in a flow and allowed to move transversely to the flow direction. Their reduced-order models are obtained in the form of (i) a single governing equation and (ii) two general coupled equations as well as the coupled lift-oscillator model. Comparisons are made with three existing models. Based on our theoretical results, and especially the reducedorder model, we conclude that the first principles development herein is a viable framework for the modeling of complex fluid-structure interaction problems such

\footnotetext{
* Corresponding author

${ }^{* *}$ Corresponding author

Email addresses: sohrob.mottaghi@faa.gov (S. Mottaghi), benaroya@rci.rutgers.edu (H. Benaroya)
}

Preprint submitted to Journal of Sound and Vibration

June 30, 2016

(C) 2016. This manuscript version is made available under the Elsevier user license http://www.elsevier.com/open-access/userlicense/1.0/ 
as vortex-induced oscillations.

Keywords: Reduced-order modeling; Fluid-structure interaction;

Vortex-induced vibration; Jourdain's Principle; Circular cylinder wakes;

Vortex shedding

\section{Introduction}

The problem of fluid-structure interaction (FSI) has long been one of the great challenges in engineering. It is a crucial consideration in the design of many engineering systems, such as offshore structures, aircraft and bridges. While the importance of the subject has been understood for well over a century, it has only been in the later part of the twentieth century that efforts have been made to analytically model the general behavior of such systems. Parallel to analytical attempts, many experiments have been devoted to gathering data and interpreting such interactions. Consequently, analytical dynamics modeling of such problems has evolved with coupling to experimental data resulting in various semi-analytical representations. Generally, attempts have been made to model vortex-induced vibration (VIV) problems by few-degree-of-freedom (DOF) oscillatory models; therefore, they are referred to as reduced-order models.

In experimental studies of VIV, certain types of structural configurations have been preferred where a rigid solid body with one or two degree(s) of freedom is immersed in a flow. While experiments have been conducted on variety of solid shapes (and occasionally on flexible bodies), reduced-order semi-analytical models have been generally developed for single DOF rigid bluff bodies, specifically for circular cylinders. The most commonly used model, here called the model problem, is a type of translating cylinder or an inverted pendulum that is immersed in a flow, resting on elastic supports, and only allowed to move transversely to the flow direction [1. Schematic diagrams of the two representative configurations of the model problem are shown in Fig. 1 .

The model problem has been widely used since it possesses a simple geometric 


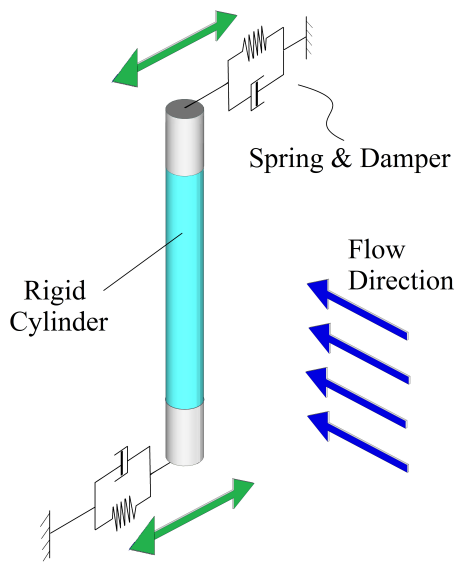

(a)

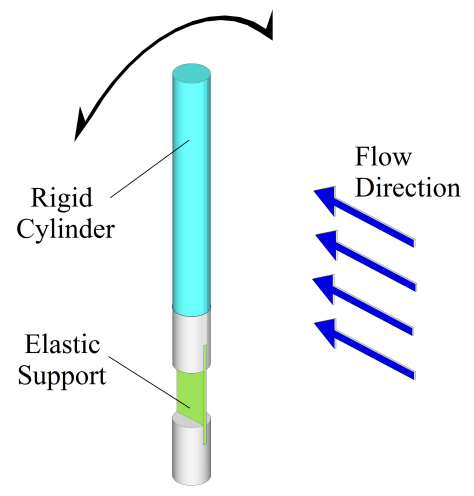

(b)

Figure 1: Representative configuration of the model problem: (a) translating cylinder, and (b) inverted pendulum.

configuration, and yet, it exhibits the majority of the nonlinear behaviors of VIV systems. Both in experimental and analytical studies, the flow is controlled or considered to be two dimensional for all time, as are the shedding vortices. Reviews of experiments for VIV can be found in [2] and 3.

Efforts to model VIV as reduced-order systems can be divided into two categories: empirical models and first-principles models. Moreover, the empirical models can be divided into two subcategories: experimental force-coefficient models and wake-oscillator (wake-body) models.

The experimental force coefficient models are single DOF models. They only include a single forcing function obtained experimentally. The wake-oscillator models are based on the assumption that an immersed structure in a flow experiences hydrodynamic forces that are the result of nonlinear-oscillator-like systems. Therefore, the aim is to obtain nonlinear fluid force equations from the experimentally acquired data that can be coupled with the structural equation of motion (EOM). One significant example of this type of model is the lift-oscillator by Hartlen and Currie [4. They considered an instantaneous lift 

EOM, resulting in,

46 [2], 6] and [7]. (see Section 10).

coefficient, $c_{L}$, to be a representative variable for the oscillatory lift force. A van der Pol oscillator was chosen to govern $c_{L}$ based on the nature of VIV systems. Moreover, the forcing function of the van der Pol oscillator was arbitrarily chosen to be a multiple of the structural velocity, thus coupling it to the structural

$\left\{\begin{array}{l}\ddot{x}_{r}+2 \zeta \dot{x}_{r}+x_{r}=a \omega_{0}^{2} c_{L} \\ \ddot{c}_{L}-\alpha \omega_{0} \dot{c}_{L}+\frac{\gamma}{\omega_{0}}\left(\dot{c}_{L}\right)^{3}+\omega_{0}^{2} c_{L}=\beta \dot{x}_{r}\end{array}\right.$

where $x_{r}$ is the dimensionless structural displacement, $\zeta$ is a reduced damping coefficient, $\omega_{0}$ is the dimensionless wind speed, $a$ is a known dimensionless constant, and $\alpha, \beta$, and $\gamma$ are costants to be estimated experimentally. Two of the unknown fluid parameters $(\alpha, \beta$, and $\gamma)$ must be selected in order to obtain the best fit to an experimental data set [5].

Generally, the empirical models have relative success in capturing the features of VIV. However, they neglect the dynamics of the flow by only considering the forces as they are seen by the structure. Therefore, they do not provide much understanding of the physics of the problem. These ad hoc methods are outside of the scope of this work that is focused on first principles models, specifically, using variational principles. Reviews of the empirical models can be found in

While variational principles have been known and utilized in modeling the dynamics of solid systems for well over a century, it has only been during the past few decades that effort have been devoted to applying variational methods to FSI and VIV problems. Next, we review a few such attempts that we believe are good representative works.

An important approach to modeling fluid dynamics using variational principles is by McIver [8]. He extended Hamilton's principle for problems involving fluid-structure interactions. The applicability of McIver's equation is restricted to the cases where an approximately laminar flow is internal to the structure

Along similar lines, Xing and Price 9] modified Hamilton's principle for 
nonlinear ship-water interactions. Their extension of Hamilton's principle contained the Eulerian variation of fluid velocity inside the control volume and the flux due to the Lagrangian virtual displacement of fluid particles. Xing and Price's method requires further simplifications and assumptions as it contained both Lagrangian and Eulerian variations.

In another effort, Benaroya and Wei [10] considered FSI problems where the fluid envelopes the structure. They showed that Hamilton's principle becomes a balance of energy rates when the configuration is not known at any time. In parallel with their theoretical development, Benaroya and Wei conducted a series of experiments on the VIV of a circular cylinder (Fig. 1b) in uniform flow and showed that their model is successful in modeling the VIV problem at hand [5, 10]. However, the predicted response amplitudes were influenced by the selection of control volume. Their theoretical and experimental results imply that the the classical energy equation in integral form is missing a significant system characteristic.

In a later work, Gabbai and Benaroya [1 noted that experiments on VIV show the existence of a formation region (cavity) in the vicinity of the oscillating cylinder. They proposed that a variable can be assigned to the displacement of the cavity to which the variational operator can be imposed. Their idea has greatly improved the applicability of Hamilton's principle to FSI problems, yet it faced some difficulties with regard to the virtual work done by hydrodynamic forces.

As evident from the literature, the efforts to apply Hamilton's principle to the problems of fluid-structure interaction have had some success. They have provided more insights into the dynamics of the fluid portion of FSI systems when compared with empirical models. However, these efforts still require additional assumptions regarding the fluid forcing function in order to obtain wake-oscillator type models. Specifically, the coupling mechanism between the structural EOM and that describing the fluid dynamics is the result of assumptions made for a problem at hand rather than being the result of variational operations. Consequently, there exist no general variational approaches 
for fluid-structure interactions. In our companion work [11, 12, the application of variational principles to fluid dynamics problems is explored. Among other difficulties, one main challenge identified arises in relating the variational principles in the Lagrangian frame to the Eulerian frame. It was shown that Jourdain's principle (JP) is a very effective tool to overcome some challenges faced by other variational principles. Consequently, an extension of JP was derived for modeling fluid dynamics problems and this derivation is summarized in Section 2. Then, after a discussion of the selection and implementation of the solid boundary conditions in Sections 3 and 4 . Jourdain's principle is extended further for FSI systems in Section 5. Using the extended formulation, reducedorder models in the form of a single governing equation of motion (SEOM) and nonlinear coupled equations of motion (EOM) are obtained for the model problem in Sections 6 to 8, where we also discuss our theoretical methodology for obtaining coupled EOM from the energy rate equations. Then, it is shown that the method developed in this paper can be combined with similarity methods to obtain a lift-oscillator model similar to that proposed by Hartlen and Currie. In Section 10, comparisons are made between the reduced order model proposed in this work and those proposed by McIver, Benaroya and Wei, and Hartlen and Currie. Concluding thoughts are given in Section 11.

We note that the primary purpose of our work is to develop a framework for the derivation of reduced-order models for FSI and VIV where one can link these models to first principles. This also allows us to identify the assumptions in other models, such as the Hartlen-Currie.

\section{An Extension of Jourdain's Principle for Fluid Systems}

We consider the application of variational principles for modeling fluid dynamics problems. Having reviewed the literature on the subject, one main source of difficulty is that the concept of virtual displacement (a Lagrangian concept) does not have an Eulerian counterpart, posing many challenges in the application of Hamilton's principle and Lagrange's equation to modeling fluid and FSI 
systems. This is overcome by utilizing Jourdain's variational principle(JP). The following is a short summary of our derivation in [11, 12].

Jourdain's principle is based on the dynamic equilibrium relation, and for a system of $N$ particles is given by the relation

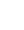

$$
\sum_{i=1}^{N}\left(m_{i} \ddot{\boldsymbol{r}}_{i}-\boldsymbol{F}_{i}\right) \cdot \delta \dot{\boldsymbol{r}}_{i}=0, \quad \text { where } \quad \delta \boldsymbol{r}_{i}=0, \delta t=0,
$$

and $m_{i}$ is the mass of particle $i$ moving on the path $\boldsymbol{r}_{i}$, vector $\boldsymbol{F}_{i}$ is the force acting on particle $i$, and $\delta \dot{\boldsymbol{r}}_{i}$ is the variation of the velocity of particle $i$ (called virtual velocity).

Alternatively, Jourdain's principle can be expressed as

7

$$
\left\{\begin{array}{c}
\frac{d}{d t}\left[\sum_{i=1}^{N} \frac{d}{d t}\left(m_{i} \dot{\boldsymbol{r}}_{i}\right) \cdot \delta \boldsymbol{r}_{i}\right]=\frac{d}{d t}\left[\sum_{i=1}^{N} \boldsymbol{F}_{i} \cdot \delta \boldsymbol{r}_{i}\right] \\
\delta t=0, \quad \delta \boldsymbol{r}_{i}=0, \quad \text { and } \quad \frac{d}{d t}\left(\delta \boldsymbol{r}_{i}\right) \neq 0
\end{array}\right.
$$

where the first line is the differentiation of d'Alembert's principle with respect to time.

In order to distinguish between Jourdain's and d'Alembert's variational operators, many authors have used the sign $\delta_{1}$ to denote Jourdain's variation. We prefer to retain the same notation $(\delta)$ with Jourdain's constraints in the remainder of this work where clarity will come from the context. That is, $\delta$ in the first line of Eq. (3) is d'Alembert's variational operator. Then, both sides of the equation are differentiated using the product rule and use is made of the $\delta \boldsymbol{r}_{i}=0$ constraint, where $\delta$ in the resulting equation is Jourdain's variational operator. Moreover, in [11, 12, Eq. (3) was used instead of Eq. (2) as it is more accessible when extending the JP for the systems of changing mass.

In order to extend Jourdain's principle, Eq. (3), the relations between the Lagrangian and the Eulerian reference frames are utilized and use is made of a mapping function $\boldsymbol{\Lambda}(\boldsymbol{x}, t)$ with the properties

$\boldsymbol{r}=\boldsymbol{\Lambda}(\boldsymbol{r}, t), \quad$ and $\quad \boldsymbol{u}(\boldsymbol{x}, t)=\frac{D}{D t} \boldsymbol{\Lambda}(\boldsymbol{x}, t)$,

where $\boldsymbol{r}$ is the Lagrangian trajectory and $\boldsymbol{u}(\boldsymbol{x}, t)$ is the Eulerian velocity of the fluid particle occupying position $\boldsymbol{x}$ at time $t$. For a control volume $C V$ 
of a Newtonian incompressible viscous fluid, we show that Jourdain's principle becomes

$$
\int_{C V(t)}\left(\rho \frac{D \boldsymbol{u}(\boldsymbol{x}, t)}{D t}-\boldsymbol{f}_{b}(\boldsymbol{x}, t)+\nabla p(\boldsymbol{x}, t)-\mu \nabla^{2} \boldsymbol{u}(\boldsymbol{x}, t)\right) \cdot \delta \boldsymbol{u}(\boldsymbol{x}, t) d V(t)=0,
$$

where $\rho$ is the density of the fluid, $D / D t$ denotes the material derivative, $\boldsymbol{f}_{b}$ represents the body force per unit volume, $p$ is the thermodynamic pressure, $\mu$ is the coefficient of dynamic viscosity, $\delta \boldsymbol{u}(\boldsymbol{x}, t)$ is Jourdain's virtual velocity imposed at the spatial position $\boldsymbol{x}$ at time $t$, and $d V(t)$ represents the differential volume element. Since $\delta \boldsymbol{u}$ is a nonzero vector, the terms of the integrand inside the parentheses must add to zero, that is, the Navier-Stokes equations are obtained.

The relation

$$
\delta \dot{L}\left(\dot{\boldsymbol{r}}_{i}, \boldsymbol{r}_{i}, t\right)=\frac{\partial \dot{L}}{\partial \dot{\boldsymbol{r}}_{i}} \cdot \delta \dot{\boldsymbol{r}}_{i}
$$

where $\dot{L}$ is the rate of Lagrangian function, is used to find energy rate equation from Jourdain principle. Since the commutation rule does not hold for the acceleration terms in the Eulerian reference frame, the non-commuting terms are extracted and the variational energy rate equation is obtained via JP to be

168

$$
\begin{aligned}
\delta \int_{C V} & \overbrace{\frac{D}{D t}\left(\frac{1}{2} \rho \boldsymbol{u} \cdot \boldsymbol{u}\right)}^{\text {rate of kinetic energy }} d V \\
& =\overbrace{\delta \int_{C S}-p \boldsymbol{u} \cdot \boldsymbol{n} d A+\delta \int_{C V}\{\mu \nabla \cdot[\nabla(\boldsymbol{u} \cdot \boldsymbol{u})-\boldsymbol{u} \times(\nabla \times \boldsymbol{u})]}^{\text {external loading }} \\
& \overbrace{-\frac{1}{2} \mu\left\{(\nabla \times \boldsymbol{u}) \cdot(\nabla \times \boldsymbol{u})+\nabla^{2}(\boldsymbol{u} \cdot \boldsymbol{u})-2\right.}^{\nabla \cdot[\boldsymbol{u} \times(\nabla \times \boldsymbol{u})]\}\} d V} d \overbrace{\delta \int_{C S} \frac{1}{2} \rho(\boldsymbol{u} \cdot \boldsymbol{u})(\boldsymbol{u} \cdot \boldsymbol{n}) d A}^{\text {non-commuting term }}
\end{aligned}
$$


173

174

175

or alternatively as,

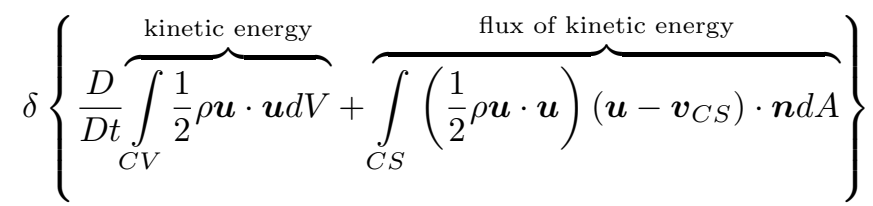

$$
\begin{aligned}
& =\delta\{\int_{C S} \overbrace{\{-p \boldsymbol{u} \cdot \boldsymbol{n}+\mu[\nabla(\boldsymbol{u} \cdot \boldsymbol{u})-\boldsymbol{u} \times(\nabla \times \boldsymbol{u})] \cdot \boldsymbol{n}}^{\text {external loading }} \\
& \overbrace{\left.-\frac{1}{2} \mu[\nabla(\boldsymbol{u} \cdot \boldsymbol{u})-2 \boldsymbol{u} \times(\nabla \times \boldsymbol{u})] \cdot \boldsymbol{n}\right\} d A-\frac{1}{2} \int_{C V} \mu(\nabla \times \boldsymbol{u}) \cdot(\nabla \times \boldsymbol{u}) d V}^{\text {viscous dissipation }} \\
& -\overbrace{\int_{C S} \frac{1}{2} \rho(\boldsymbol{u} \cdot \boldsymbol{u})(\boldsymbol{u} \cdot \boldsymbol{n}) d A}^{\text {non-commuting term }}\}
\end{aligned}
$$

where $\boldsymbol{v}_{C S}$ denotes the velocity of the control surface element $d A$, and the last integral on the right-hand side is due to the non-commuting part of acceleration term. Also, body forces have been neglected. If the only body force present is due to gravity, its potential function can be easily obtained, since gravitational forces are conservative and independent of the fluid velocity field. Eqs. (7) and (8) represent the energy rate equation in a variational format. In order to obtain the actual energy rate equation, the integrals labeled as "viscous dissipation" must be multiplied by a factor of two, since they are Rayleigh's dissipation function, $\phi$, and are obtained from the relation

$$
\delta \phi=\nabla_{\boldsymbol{u}} \phi \cdot \delta \boldsymbol{u}
$$

where $\nabla_{\boldsymbol{u}} \phi$ denotes the differentiation of $\phi$ with respect to $\boldsymbol{u}$ in the direction of $\boldsymbol{u}$.

Eqs. (7) and (8) can both be used in modeling fluid dynamic systems and FSI. Their advantages over one another mainly rely on the choice of the control volume and how the boundary conditions are implemented.

Modeling the dynamics of a system of fluid particles, alone, is a very challenging problem due to the nonlinear behavior of fluids. For fluid-structure inter- 
action (FSI) problems, the difficulties increase since the nature of the boundary conditions between fluid and structure is not fully understood, and are generally just assumptions backed by experimental results. Therefore, a discussion of boundary conditions is necessary next before we expand Eqs. (7) and (8) to FSI systems.

\section{Boundary Conditions at the Surface of Solids}

From the time of Newton until the early 20th century, one topic of intensive discussion has been the boundary conditions on solid surfaces that are interacting with viscous fluid particles [13. During the past century, many experiments were conducted and compared to the theoretical and numerical results using the no-slip condition, resulting in an almost unified agreement on the no-slip condition [14]. Yet, the no-slip condition remains an assumption (for fluid systems) since it cannot be proven using a first principles approach. The general understanding is that some intermolecular interactions result in zero relative velocity, $\boldsymbol{u}_{r}$, of fluid particles at a solid surface [15, pp. 295-296], that is,

$$
\boldsymbol{u}_{r}=\boldsymbol{u}-\boldsymbol{v}=\mathbf{0}
$$

where $\boldsymbol{u}$ is the velocity of the fluid at the surface of a solid moving with the velocity $\boldsymbol{v}$.

Alternatively, another definition of the no-slip condition is that the tangential components of the relative velocity must vanish [16. This condition must be considered together with the no penetration condition for the normal direction, that is, no fluid particles can penetrate the solid.

While the no-slip condition seems to be compatible with many experimental observations, the wetting property of the surface, the velocity of the flow, surface roughness and gas bubbles are among the factors shown to affect the no-slip boundary condition [17, 18, 19, 20, More discussion can be found in [14, p. 1222].

The applicability of the no-slip condition, or any other condition, must be justified by a comparison with experimental observations. The no-slip condition, 
in its most complete form as expressed by Eq. (10), satisfies the no-penetration conditions as well. Therefore, for reduced-order models, care must be exercised so that the reduced number of equations can capture the main characteristics of the FSI. For example, if the no-slip condition is strongly imposed, then the resulting reduced-order model most likely cannot represent the separation and shedding of the vortices from the solid structure, unless additional assumptions are made. Consequently, it might be necessary to relax the no-slip condition to some extent in analytical or numerical modeling. In fact, the no-slip condition has not been applied in its most complete form in many computational simulations or analytical models of FSI. It is implicitly implemented, for example, via other constraints in the system [21] or through an assumed force field [22]. Moreover, the no-slip condition is often explicitly implemented and is then relaxed or corrected in some later steps in the modeling process [23]. Alternatively, some works weakly impose the no-slip condition [16].

For reduced-order models, the difficulties increase since they are less flexible with respect to the choice of boundary conditions. We continue our derivation by considering the no-slip boundary condition as it is widely accepted in the literature. We will also define explicit and implicit no-slip conditions.

The control volume of interest is described next.

\section{Control Volume Definition}

The derived variational formulations, Eqs. (7) and (8), did not face any restrictions in the selection of the control volume. Regarding FSI and VIV problems, the methodology we use later imposes a limitation on the selection the $C V$ in that some portions of the control surface must be comprised of solid surfaces. Since no fluid can cross the solid surface, these control surfaces are referred to as closed control surfaces, $C S_{C}$. The other surfaces, where fluid can cross, are called open control surfaces, $C S_{O}$.

Since we are interested in reduced-order modeling of the model problem (Fig. 1), we select the control volume shown in Fig. 2 and assume that both the 


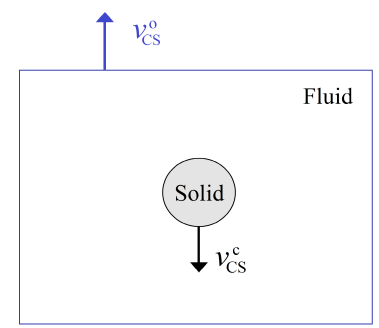

Figure 2: Selected control volume; rectangular and circular control surfaces can move independently.

rectangular and circular control surfaces move rigidly and independently of each other. The shape of the open portion of the control volume is selected to be rectangular for simplicity. While our efforts in this paper are to derive the EOM for a single DOF rigid body coupled to the fluid oscillator, the same method can be used for modeling a general deformable solid as well.

Fig. 2 should be interpreted as a three-dimensional volume where the third dimension is into the paper and of arbitrary depth.

So far, we have not considered any solid body in our derivations. In order to include a solid structure, we start by considering the original statement of Jourdain's principle for a system volume (material volume).

\section{Extended JP for FSI Systems}

Jourdain's variational principle for a set of $N$ fluid and solid particles, assuming that the system consists of $M$ fluid particles where $M<N$, is

$264 \quad\left\{\begin{array}{l}\frac{d}{d t}\left[\sum_{i=1}^{M}\left(m_{i} \ddot{\boldsymbol{r}}_{i}-\boldsymbol{F}_{i}\right) \cdot \delta \boldsymbol{r}_{i}+\sum_{j=M+1}^{N}\left(m_{j} \ddot{\boldsymbol{r}}_{j}-\boldsymbol{F}_{j}\right) \cdot \delta \boldsymbol{r}_{j}\right]=0 \\ \delta t=0, \quad \delta \boldsymbol{r}_{i}=0, \quad \delta \boldsymbol{r}_{j}=0, \quad \frac{d}{d t}\left(\delta \boldsymbol{r}_{i}\right) \neq 0, \quad \text { and } \quad \frac{d}{d t}\left(\delta \boldsymbol{r}_{j}\right) \neq 0 .\end{array}\right.$ 
If the set of particles is continuous during some time interval, then Eq. 111 can be expressed in integral form as

$$
\left\{\begin{array}{r}
\frac{d}{d t} \int_{V_{f}}\left[\rho \frac{d \boldsymbol{u}(\boldsymbol{r}, t)}{d t}-\boldsymbol{f}(\boldsymbol{r}, t)\right] \cdot \delta \boldsymbol{\Lambda}(\boldsymbol{r}, t) d V_{f} \\
\quad+\frac{d}{d t} \int_{V_{s}}\left[\rho_{s} \frac{d \dot{\boldsymbol{r}}_{s}}{d t}-\boldsymbol{f}_{s}\left(\boldsymbol{r}_{s}\right)\right] \cdot \delta \boldsymbol{r}_{s} d V_{s}-\frac{d}{d t} \int_{\substack{\text { Solid } \\
\text { Surface }}} \boldsymbol{f}_{s}^{*} \cdot \delta \boldsymbol{r}_{s} d A_{s}=0 \\
\delta t=0, \quad \delta \boldsymbol{\Lambda}(\boldsymbol{r}, t)=0, \quad \delta \boldsymbol{r}_{s}=0, \quad \frac{d}{d t}[\delta \boldsymbol{\Lambda}(\boldsymbol{r}, t)] \neq 0, \quad \text { and } \frac{d}{d t}\left(\delta \boldsymbol{r}_{s}\right) \neq 0,
\end{array}\right.
$$

where $V_{f}$ is the material volume of the fluid particles, $V_{s}$ is the material volume of solid particles, $\rho_{s}$ denotes the density of solid, $\boldsymbol{r}_{s}$ is the Lagrangian position of the solid particles, $\boldsymbol{f}_{s}\left(\boldsymbol{r}_{s}\right)$ denotes the force density (force per unit volume) in the solid domain at $\boldsymbol{r}_{s}, \boldsymbol{f}_{s}^{*}$ is the density of the fluid forces acting on the solid surface, $\boldsymbol{r}$ is the Lagrangian position of the fluid particles, and $\boldsymbol{\Lambda}$ is the mapping function introduced earlier. Note that $\boldsymbol{f}_{s}\left(\boldsymbol{r}_{s}\right)$ does not include any force due to the fluid dynamics. No constraints are included in deriving Eq. (12), except those imposed by Jourdain's principle. The mapping function $\boldsymbol{\Lambda}(\boldsymbol{r}, t)$ is introduced so that Lagrangian variables can be related to Eulerian variables.

For an incompressible fluid, using Eq. (5), Eq. (12) becomes

$$
\left\{\begin{array}{c}
\int_{C V(t)}\left(\rho \frac{D \boldsymbol{u}(\boldsymbol{x}, t)}{D t}-\boldsymbol{f}_{b}(\boldsymbol{x}, t)+\nabla p(\boldsymbol{x}, t)-\mu \nabla^{2} \boldsymbol{u}(\boldsymbol{x}, t)\right) \cdot \delta \boldsymbol{u}(\boldsymbol{x}, t) d V(t) \\
+\frac{d}{d t} \int_{V_{s}}\left[\rho_{s} \frac{d \dot{\boldsymbol{r}}_{s}}{d t}-\boldsymbol{f}_{s}\left(\boldsymbol{r}_{s}\right)\right] \cdot \delta \boldsymbol{r}_{s} d V_{s}-\frac{d}{d t} \int_{\substack{\text { Solid } \\
\text { Surface }}} \boldsymbol{f}_{s}^{*} \cdot \delta \boldsymbol{r}_{s} d A_{s}=0 \\
\delta t=0, \quad \delta \boldsymbol{r}_{s}=0, \quad \text { and } \quad \frac{d}{d t}\left(\delta \boldsymbol{r}_{s}\right) \neq 0
\end{array}\right.
$$

The energy rate equation for a general control volume of fluid particles was 
found in Eqs. (7) and (8). Introducing Eq. (8) into Eq. (13) results in

$$
\begin{aligned}
& \delta\left\{\frac{D}{D t} \int_{C V} \frac{1}{2} \rho \boldsymbol{u} \cdot \boldsymbol{u} d V+\int_{C S}\left(\frac{1}{2} \rho \boldsymbol{u} \cdot \boldsymbol{u}\right)\left(\boldsymbol{u}-\boldsymbol{v}_{C S}\right) \cdot \boldsymbol{n} d A\right. \\
& \overbrace{-\int_{C S}\{-p \boldsymbol{u} \cdot \boldsymbol{n}+\mu[\nabla(\boldsymbol{u} \cdot \boldsymbol{u})-\boldsymbol{u} \times(\nabla \times \boldsymbol{u})] \cdot \boldsymbol{n}}^{\text {viscous dissipation }} \\
& \overbrace{\left.-\frac{1}{2} \mu[\nabla(\boldsymbol{u} \cdot \boldsymbol{u})-2 \boldsymbol{u} \times(\nabla \times \boldsymbol{u})] \cdot \boldsymbol{n}\right\} d A+\frac{1}{2} \int_{C V} \mu(\nabla \times \boldsymbol{u}) \cdot(\nabla \times \boldsymbol{u}) d V} \\
& \text { non-commuting term } \\
& \overbrace{\left.\int_{C S} \frac{1}{2} \rho(\boldsymbol{u} \cdot \boldsymbol{u})(\boldsymbol{u} \cdot \boldsymbol{n}) d A\right\}} \\
& +\frac{d}{d t} \int_{V_{s}}\left[\rho_{s} \frac{d \dot{\boldsymbol{r}}_{s}}{d t}-\boldsymbol{f}_{s}\left(\boldsymbol{r}_{s}\right)\right] \cdot \delta \boldsymbol{r}_{s} d V_{s}-\frac{d}{d t} \int_{\substack{\text { Solid } \\
\text { Surface }}} \boldsymbol{f}_{s}^{*} \cdot \delta \boldsymbol{r}_{s} d A_{s}=0 \\
& \delta t=0, \quad \delta \boldsymbol{r}_{s}=0, \quad \text { and } \quad \frac{d}{d t}\left(\delta \boldsymbol{r}_{s}\right) \neq 0
\end{aligned}
$$

where body forces are neglected for fluid particles.

Eq. 14 represents a general variational formulation for a control volume of a Newtonian incompressible viscous fluid and a general solid body. In order to specialize this equation further, boundary conditions must be considered and the stress-strain relations are required for elastic solid bodies. If the structure is assumed to be rigid, such as the model problem, forces acting at the elastic boundaries must be known. Therefore, we continue by considering the model problem to illustrate the required procedure. In the following section, Eq. 14 is modified further to obtain a single governing EOM describing the dynamics of the FSI system.

\section{Modeling FSI: Single Governing EOM}

In deriving Eq. (14) we have not considered the boundary conditions on the solid surface. Therefore, we continue our derivation by considering the no-slip condition in its most complete form. 
To include the no-slip condition, we consider the second line of Eq. (14), the fluid pressure and shear forces, and divide these into integrals over the closed and the open portions of the control surface,

$\delta \int_{C S}\{-p \boldsymbol{u} \cdot \boldsymbol{n}+\mu[\nabla(\boldsymbol{u} \cdot \boldsymbol{u})-\boldsymbol{u} \times(\nabla \times \boldsymbol{u})] \cdot \boldsymbol{n}\} d A=\delta \int_{C S_{C}} \ldots d A+\delta \int_{C S_{O}} \ldots d A$

Combining the no-slip condition with Jourdain's principle requirement for zero virtual displacement, requires the particles at the solid surface remain in the same relative position. The shear terms of Eq. (15) (terms containing $\mu$ ) are representative of external loads and are not dissipative. Therefore, the power lost by the structure must be absorbed by the fluid and vice versa at only the closed control surface. The virtual velocities must be compatible with system's constraints, thus $\delta \dot{\boldsymbol{r}}_{s}=\delta \boldsymbol{u}$ at the closed control surface.

Therefore, the forces acting on the fluid by the solid surface must be equal and opposite to $\boldsymbol{f}_{s}^{*}$. They are action and reaction forces at all points on the solid surface, that is,

$$
\frac{d}{d t} \int_{\substack{\text { Solid } \\ \text { Surface }}} \boldsymbol{f}_{s}^{*} \cdot \delta \boldsymbol{r}_{s} d A_{s}=-\delta \int_{C S_{C}}\{-p \boldsymbol{u} \cdot \boldsymbol{n}+\mu[\nabla(\boldsymbol{u} \cdot \boldsymbol{u})-\boldsymbol{u} \times(\nabla \times \boldsymbol{u})] \cdot \boldsymbol{n}\} d A .
$$

Substituting Eq. (16) into Eq. (15), and introducing it into Eq. (14), we obtain 


$$
\begin{aligned}
& \int \delta \frac{D}{D t} \int_{C V} \frac{1}{2} \rho \boldsymbol{u} \cdot \boldsymbol{u} d V+\int_{\substack{C S \\
\text { loading external to the fluid }}}\left(\frac{1}{2} \rho \boldsymbol{u} \cdot \boldsymbol{u}\right)\left(\boldsymbol{u}-\boldsymbol{v}_{C S}\right) \cdot \boldsymbol{n} d A \\
& \overbrace{-\int_{C S_{O}}\{-p \boldsymbol{u} \cdot \boldsymbol{n}+\mu[\nabla(\boldsymbol{u} \cdot \boldsymbol{u})-\boldsymbol{u} \times(\nabla \times \boldsymbol{u})] \cdot \boldsymbol{n}\} d A} \\
& \{\overbrace{+\int_{C S} \frac{1}{2} \mu[\nabla(\boldsymbol{u} \cdot \boldsymbol{u})-2 \boldsymbol{u} \times(\nabla \times \boldsymbol{u})] \cdot \boldsymbol{n} d A+\frac{1}{2} \int_{C V} \mu(\nabla \times \boldsymbol{u}) \cdot(\nabla \times \boldsymbol{u}) d V}^{\text {viscous dissipation }} \\
& \overbrace{\left.\int_{C S} \frac{1}{2} \rho(\boldsymbol{u} \cdot \boldsymbol{u})(\boldsymbol{u} \cdot \boldsymbol{n}) d A\right\}} \\
& +\frac{d}{d t} \int_{V_{s}}\left[\rho_{s} \frac{d \dot{\boldsymbol{r}}_{s}}{d t}-\boldsymbol{f}_{s}\left(\boldsymbol{r}_{s}\right)\right] \cdot \delta \boldsymbol{r}_{s} d V_{s}=0 \\
& \delta t=0, \quad \delta \boldsymbol{r}_{s}=0, \quad \text { and } \quad \frac{d}{d t}\left(\delta \boldsymbol{r}_{s}\right) \neq 0 \text {. }
\end{aligned}
$$

Eq. (17) is valid for any control volume of a Newtonian incompressible viscous fluid and any solid body (rigid or deformable). We next continue our derivation for the model problem which assumes the solid body to be rigid. For a deformable solid body, similar manipulations need to be performed, given the stress-strain relations $\boldsymbol{f}_{s}\left(\boldsymbol{r}_{s}, t\right)$.

\subsection{Single Governing EOM for the Translating Cylinder}

Consider the model problem in the form of the translating cylinder shown in Fig. 1a. One generalized coordinate is required to fully describe the motion of the cylinder. Let the generalized coordinate be the Lagrangian coordinate $\boldsymbol{x}_{s}(t)$ measured from the center of the cylinder when it is at rest, perpendicular to the axis of symmetry of the cylinder and transverse to the flow direction.

Setting $\boldsymbol{r}_{s}(t)=\boldsymbol{x}_{s}(t)$ and $\boldsymbol{f}_{s}\left(\boldsymbol{x}_{s}\right)=\frac{1}{V_{s}}\left(-C \dot{\boldsymbol{x}}_{s}-k \boldsymbol{x}_{s}\right)$ in Eq. [17), the 
corresponding terms can be replaced as follows,

$$
\begin{aligned}
\frac{d}{d t} \int_{V_{s}}\left[\rho_{s} \frac{d \dot{\boldsymbol{r}}_{s}}{d t}-\boldsymbol{f}_{s}\left(\boldsymbol{r}_{s}\right)\right] \cdot \delta \boldsymbol{r}_{s} d V_{s} & =\frac{d}{d t}\left[\left(m_{s} \ddot{\boldsymbol{x}}_{s}+C \dot{\boldsymbol{x}}_{s}+k \boldsymbol{x}_{s}\right) \cdot \delta \boldsymbol{x}_{s}\right] \\
& =\left(m_{s} \ddot{\boldsymbol{x}}_{s}+C \dot{\boldsymbol{x}}_{s}+k \boldsymbol{x}_{s}\right) \cdot \delta \dot{\boldsymbol{x}}_{s},
\end{aligned}
$$

where $C$ is the net support damping constant and $k$ denotes the net support spring constant. In deriving Eq. (18), the structural terms are differentiated and the variational constraint $\left(\delta \boldsymbol{x}_{s}=0\right)$ is imposed. Also, the rigidity of the cylinder is considered in the integration over the solid volume.

Eq. (6) can be used to obtain the energy rate equation for the conservative structural terms,

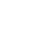

$$
\left(m_{s} \ddot{\boldsymbol{x}}_{s}+k \boldsymbol{x}_{s}\right) \cdot \delta \dot{\boldsymbol{x}}_{s}=\delta\left(m_{s} \ddot{\boldsymbol{x}}_{s} \cdot \dot{\boldsymbol{x}}_{s}+k \boldsymbol{x}_{s} \cdot \dot{\boldsymbol{x}}_{s}\right) .
$$

For the nonconservative linear damper, we use Rayleigh's dissipation function,

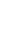

$$
\delta\left(\frac{1}{2} C \dot{\boldsymbol{x}}_{s} \cdot \dot{\boldsymbol{x}}_{s}\right)=C \dot{\boldsymbol{x}}_{s} \cdot \delta \dot{\boldsymbol{x}}_{s}
$$

In deriving Eq. (17), we did not add any constraints with regard to the nature of solid-fluid interaction other than action and reaction forces. In order to include the boundary conditions, we separate the integrals over the control surface into integrals over its open and closed portions. For the flux of kinetic energy, we have

$$
\begin{aligned}
& \int_{C S}\left(\frac{1}{2} \rho \boldsymbol{u} \cdot \boldsymbol{u}\right)\left(\boldsymbol{u}-\boldsymbol{v}_{C S}\right) \cdot \boldsymbol{n} d A \\
& =\int_{C S_{C}}\left(\frac{1}{2} \rho \boldsymbol{u} \cdot \boldsymbol{u}\right)\left(\boldsymbol{u}-\boldsymbol{v}_{C S}^{c}\right) \cdot \boldsymbol{n} d A+\int_{C S_{O}}\left(\frac{1}{2} \rho \boldsymbol{u} \cdot \boldsymbol{u}\right)\left(\boldsymbol{u}-\boldsymbol{v}_{C S}^{o}\right) \cdot \boldsymbol{n} d A,
\end{aligned}
$$

where $\boldsymbol{v}_{C S}^{o}$ and $\boldsymbol{v}_{C S}^{c}$ are the velocities of the open and closed portions of the control surface, respectively. The first integral on the right-hand side of Eq. (21) is equal to zero due to the no-penetration condition.

For the fluid dissipative forces at the control surfaces, we expand the fourth integral in Eq. 17] as follows

$$
\int_{C S} \frac{1}{2} \mu[\nabla(\boldsymbol{u} \cdot \boldsymbol{u})-2 \boldsymbol{u} \times(\nabla \times \boldsymbol{u})] \cdot \boldsymbol{n} d A=\int_{C S_{O}} \ldots d A+\int_{C S_{C}} \ldots d A \text {. }
$$


The second integral, over the closed control surface, on the right hand side is interpreted in the following way. We expect energy to be dissipated at higher rates near the structure due to a larger velocity gradient. Even though the no-slip condition implies that no energy is lost due to friction because of zero relative motion between the fluid and solid, viscous dissipation can be envisioned between the fluid particles at the solid surface and those adjacent to them. So, we choose to keep the dissipative terms at the closed control surfaces and continue our derivations.

Finally, for the non-commuting terms of Eq. (17), we also consider the noslip condition that states that at the closed control surface $\boldsymbol{u}=\dot{\boldsymbol{x}}_{s}$ for all time. Replacing $\boldsymbol{u}$ by $\dot{\boldsymbol{x}}_{s}$ at the closed surfaces and considering that the commutation rule holds for $\dot{\boldsymbol{x}}_{s}$ (because it is Lagrangian), the non-commuting terms disappear. Therefore, in Eq. 17] we have

$\int_{C S} \frac{1}{2} \rho(\boldsymbol{u} \cdot \boldsymbol{u})(\boldsymbol{u} \cdot \boldsymbol{n}) d A=\int_{C S_{O}} \frac{1}{2} \rho(\boldsymbol{u} \cdot \boldsymbol{u})(\boldsymbol{u} \cdot \boldsymbol{n}) d A$

Having specified the boundary condition, the energy rate equation for the translating cylinder problem is obtained by substituting Eqs. 119 and (20) into Eq. 18 for the structural terms, and introducing the resulting relation 
355

372

together with Eqs. (21), (22) and (23) into Eq. (17), resulting in

$$
\begin{array}{r}
\delta\left\{\frac{D}{D t} \int_{C V} \frac{1}{2} \rho \boldsymbol{u} \cdot \boldsymbol{u} d V+\int_{C S_{O}}\left(\frac{1}{2} \rho \boldsymbol{u} \cdot \boldsymbol{u}\right)\left(\boldsymbol{u}-\boldsymbol{v}_{C S}^{o}\right) \cdot \boldsymbol{n} d A\right. \\
-\int_{C S_{O}} \overbrace{\{-p \boldsymbol{u} \cdot \boldsymbol{n}+\mu[\nabla(\boldsymbol{u} \cdot \boldsymbol{u})-\boldsymbol{u} \times(\nabla \times \boldsymbol{u})] \cdot \boldsymbol{n}}^{\text {external load at open CS }}
\end{array}
$$

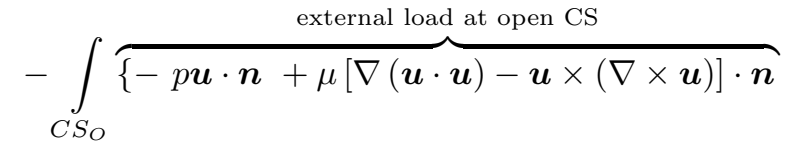

viscous dissipation at open CS

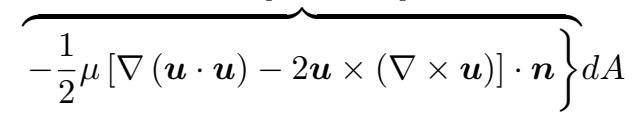

viscous dissipation at solid surface

$$
+\overbrace{\int_{C S_{C}} \frac{1}{2} \mu[\nabla(\boldsymbol{u} \cdot \boldsymbol{u})-2 \boldsymbol{u} \times(\nabla \times \boldsymbol{u})] \cdot \boldsymbol{n} d A}
$$

$$
\begin{array}{r}
+\overbrace{\int_{C V} \frac{1}{2} \mu(\nabla \times \boldsymbol{u}) \cdot(\nabla \times \boldsymbol{u}) d V}^{\text {viscous dissipation inside the } \mathrm{CV}}+\int_{C S_{O}} \frac{1}{2} \rho(\boldsymbol{u} \cdot \boldsymbol{u})(\boldsymbol{u} \cdot \boldsymbol{n}) d A \\
\left.+m_{s} \ddot{\boldsymbol{x}}_{s} \cdot \dot{\boldsymbol{x}}_{s}+\frac{1}{2} C \dot{\boldsymbol{x}}_{s} \cdot \dot{\boldsymbol{x}}_{s}+k \boldsymbol{x}_{s} \cdot \dot{\boldsymbol{x}}_{s}\right\}=0,
\end{array}
$$

where the fluid parameters are expressed in an Eulerian reference frame and the structural terms are Lagrangian. We note that, unlike d'Alembert's variational operator, Jourdain's variational operator is the same in both the Lagrangian and the Eulerian frames [11, 12].

Rayleigh's dissipation function must be multiplied by a factor of two in order to obtain the energy rate equation 24]. Therefore, the energy rate equation for the translating cylinder problem is

30

$$
\frac{D}{D t} \int_{C V} \frac{1}{2} \rho \boldsymbol{u} \cdot \boldsymbol{u} d V+\int_{C S_{O}}\left(\frac{1}{2} \rho \boldsymbol{u} \cdot \boldsymbol{u}\right)\left(\boldsymbol{u}-\boldsymbol{v}_{C S}^{o}\right) \cdot \boldsymbol{n} d A
$$

${ }_{C S_{O}}-\int_{C S_{C}}[-p \boldsymbol{u} \cdot \boldsymbol{n}+\mu \boldsymbol{u} \times(\nabla \times \boldsymbol{u}) \cdot \boldsymbol{n}] d A+\int_{C} \mu[\nabla(\boldsymbol{u} \cdot \boldsymbol{u})-2 \boldsymbol{u} \times(\nabla \times \boldsymbol{u})] \cdot \boldsymbol{n} d A$

$$
+\int_{C V} \mu(\nabla \times \boldsymbol{u}) \cdot(\nabla \times \boldsymbol{u}) d V+\int_{C S_{O}} \frac{1}{2} \rho(\boldsymbol{u} \cdot \boldsymbol{u})(\boldsymbol{u} \cdot \boldsymbol{n}) d A
$$

$$
+m_{s} \ddot{\boldsymbol{x}}_{s} \cdot \dot{\boldsymbol{x}}_{s}+C \dot{\boldsymbol{x}}_{s} \cdot \dot{\boldsymbol{x}}_{s}+k \boldsymbol{x}_{s} \cdot \dot{\boldsymbol{x}}_{s}=0,
$$


where we summed the viscous terms at open control surface.

The model problem in the form of inverted pendulum is considered in the following section.

\subsection{Single Governing EOM for the Inverted Pendulum}

Similar to the translating cylinder, the inverted rigid pendulum (Fig. 1b) is a single DOF structure with generalized coordinate the angle of rotation $\theta$. As per Benaroya and Wei [10, we assume that three-dimensional effects can be ignored (vortices remain two-dimensional), weight and buoyancy forces must be included for the structure, and the resultant of all the forces act at the geometric center of the circular cylinder. Moreover, the horizontal plane passing through the center of geometry contains the two dimensional vortices.

Therefore, by replacing the last term in Eq. (17) by

$$
\left[I_{0} \ddot{\theta}+C_{T} \dot{\theta}+k_{T} \theta-\left(m_{s} g-B\right) \frac{L}{2} \sin \theta \mathbf{e}_{\theta}\right] \cdot \delta \theta \mathbf{e}_{\theta},
$$

the energy rate equation is found to be

$$
\frac{D}{D t} \int_{C V} \frac{1}{2} \rho \boldsymbol{u} \cdot \boldsymbol{u} d V+\int_{C S_{O}}\left(\frac{1}{2} \rho \boldsymbol{u} \cdot \boldsymbol{u}\right)\left(\boldsymbol{u}-\boldsymbol{v}_{C S}^{o}\right) \cdot \boldsymbol{n} d A
$$

$-\int_{C S_{O}}[-p \boldsymbol{u} \cdot \boldsymbol{n}+\mu \boldsymbol{u} \times(\nabla \times \boldsymbol{u}) \cdot \boldsymbol{n}] d A+\int_{C S_{C}} \mu[\nabla(\boldsymbol{u} \cdot \boldsymbol{u})-2 \boldsymbol{u} \times(\nabla \times \boldsymbol{u})] \cdot \boldsymbol{n} d A$

$$
\begin{aligned}
+\int_{C V} \mu & (\nabla \times \boldsymbol{u}) \cdot(\nabla \times \boldsymbol{u}) d V+\int_{C S_{O}} \frac{1}{2} \rho(\boldsymbol{u} \cdot \boldsymbol{u})(\boldsymbol{u} \cdot \boldsymbol{n}) d A \\
& \left.+I_{0} \ddot{\theta} \dot{\theta}+C_{T} \dot{\theta}^{2}+k_{T} \dot{\theta}-\left[\left(m_{s} g-B\right) \frac{L}{2} \sin \theta\right] \dot{\theta}\right\}=0 .
\end{aligned}
$$

The derivations required to obtain this equation are not presented here since they are similar to those shown in the last section. Detailed derivations can be found in $[12$.

Having obtained a single degree-of-freedom (SDOF) equation for the model problem, we next seek reduced-order models that are in the form of two coupled EOM. We begin by explaining our methodology based on the fundamental concepts of the variational calculus. 


\section{Coupled Equations of Motion: Fundamental Variational Basis}

Having obtained the variational energy rate equations, $\delta \dot{L}=0$, (Eq. 24 , and the variational equation corresponding to Eq. (26)), we wish to obtain two nonlinear coupled equations that can fully describe the main characteristics of the dynamics of a FSI system.

In analytical mechanics, the partial derivatives of the Lagrangian function with respect to velocities results in a fundamental concept called the generalized momenta [25], $p_{i}$, defined as

$$
p_{i}=\frac{\partial L}{\partial \dot{q}_{i}},
$$

where $\dot{q}_{i}$ is the generalized velocity that can be selected to be either the Lagrangian or the Eulerian velocities.

The following derivations in many ways are similar to that concept as a result of the definition of Jourdain's variational operator [11, 12,

$$
\delta \psi=\lim _{\varepsilon \rightarrow 0} \frac{1}{\varepsilon}[\psi(\boldsymbol{u}+\varepsilon \delta \boldsymbol{u})-\psi(\boldsymbol{u})],
$$

and

$$
\delta \psi=\frac{\partial \psi}{\partial \boldsymbol{u}} \cdot \delta \boldsymbol{u},
$$

where $\psi$ is a scalar function, $\boldsymbol{u}$ is the velocity field and $\delta \boldsymbol{u}$ is the variation of the velocity field. However, here we are dealing with the rate of the Lagrangian function, $\dot{L}$ (or in the Eulerian reference frame, $D L / D t$ ), instead of the Lagrangian $L$, which results in the rate of generalized momenta.

A fundamental concept of variational calculus, called Noether's principle, states that any infinitesimal transformation of either the action variables, or the independent variables, involving a constant parameter results in a certain conservation law if the Lagrangian remains unchanged [25]. In [11, 12, we have shown that the energy rate equation $(\dot{L}=0)$ can be directly obtained from Jourdain's principle using Eq. (29). That is, the corresponding differential equations can be obtained by using the definition of Jourdain's principle and by choosing $\boldsymbol{u}$ and $\dot{\boldsymbol{r}}_{s}$ to be the independent generalized velocities. Thus,

$$
\delta \dot{L}=\frac{\partial \dot{L}}{\partial \boldsymbol{u}} \cdot \delta \boldsymbol{u}+\frac{\partial \dot{L}}{\partial \dot{\boldsymbol{r}}_{s}} \cdot \delta \dot{\boldsymbol{r}}_{s}=0
$$


where $\partial \dot{L} / \partial \boldsymbol{u}$ and $\partial \dot{L} / \partial \dot{\boldsymbol{r}}_{s}$ are obtained using Eqs. 28) and (29).

Since $\delta \boldsymbol{u}$ and $\delta \dot{\boldsymbol{r}}_{s}$ are arbitrary, non-zero vectors, Equation 30 results in

$$
\begin{aligned}
\frac{\partial \dot{L}}{\partial \boldsymbol{u}} & =0, \\
\frac{\partial \dot{L}}{\partial \dot{\boldsymbol{r}}_{s}} & =0 .
\end{aligned}
$$

For the model problem, these are four coupled equations: Eq. 32 is a single differential equation (since the structure has a single DOF) representing the solid structure and Eq. (31) represents three differential equations describing the flow.

The partial scalar function $\delta \dot{L}_{\boldsymbol{u}}$

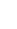

$$
\delta \dot{L}_{\boldsymbol{u}}=\frac{\partial \dot{L}}{\partial \boldsymbol{u}} \cdot \delta \boldsymbol{u}
$$

also equals zero, implying that $\dot{L}_{\boldsymbol{u}}=0$, which is an integral power equation governing the fluid dynamics coupled to the structural EOM, $\partial \dot{L} / \partial \dot{\boldsymbol{r}}_{s}=0$.

Generally, variational methods are powerful tools for conservative systems. The approach explained here requires monogenic forces and a scleronomic system. Frictional forces (structural damping and viscous forces) are polygenic and the corresponding energy rate terms are obtained using Rayleigh's dissipation function.

\section{Coupled Equations of Motion: The Wake Oscillator}

In this section, we wish to obtain reduced-order wake-oscillator models comprised of two coupled EOM using the methodology explained in the previous section. In Section 3, we discussed that imposing the no-slip condition in its most complete form may weaken the reduced order model's ability to capture the true dynamics of the FSI system, and that weak forms of no-slip conditions have generally been applied in many computational simulations or analytical models of FSI. For wake-oscillator models, the boundary conditions are extremely important as these essentially determine the nature of the coupling between the two governing equations of motion. 

pressed as

467

In deriving Eq. $(17), \boldsymbol{f}_{s}^{*} \cdot \delta \boldsymbol{r}_{s}$ was shown to represent the boundary condition on the solid surface. Therefore, we start the modeling process from the variational formulation expressed by Eq. (14), and neglect $\boldsymbol{f}_{s}^{*} \cdot \delta \boldsymbol{r}_{s}$ in order to introduce it explicitly and implicitly in the following two sections, where this terminology will become clear.

For simplicity, only the model problem in the form of the translating cylinder is considered. We apply Gauss' divergence theorem to Eq. (14) in order to take the non-commuting term inside the control volume and substitute the structural terms from Eqs. (19) and 20, resulting in

$$
\delta\left\{\frac{D}{D t} \int_{C V} \frac{1}{2} \rho \boldsymbol{u} \cdot \boldsymbol{u} d V+\int_{C S}\left(\frac{1}{2} \rho \boldsymbol{u} \cdot \boldsymbol{u}\right)\left(\boldsymbol{u}-\boldsymbol{v}_{C S}\right) \cdot \boldsymbol{n} d A\right.
$$

loading external to the fluid

$$
\overbrace{-\int_{C S}\{-p \boldsymbol{u} \cdot \boldsymbol{n}+\mu[\nabla(\boldsymbol{u} \cdot \boldsymbol{u})-\boldsymbol{u} \times(\nabla \times \boldsymbol{u})] \cdot \boldsymbol{n}}
$$

$$
\overbrace{\left.-\frac{1}{2} \mu[\nabla(\boldsymbol{u} \cdot \boldsymbol{u})-2 \boldsymbol{u} \times(\nabla \times \boldsymbol{u})] \cdot \boldsymbol{n}\right\} d A+\frac{1}{2} \int_{C V} \mu(\nabla \times \boldsymbol{u}) \cdot(\nabla \times \boldsymbol{u}) d V}^{\text {viscous dissipation }}
$$

$$
\begin{aligned}
& +\overbrace{\int_{C V} \nabla \cdot\left[\frac{1}{2} \rho(\boldsymbol{u} \cdot \boldsymbol{u}) \boldsymbol{u}\right] d V}^{\text {non-commuting term }}\} \\
& +\delta\left(m_{s} \ddot{\boldsymbol{x}}_{s} \cdot \dot{\boldsymbol{x}}_{s}+\frac{1}{2} C \dot{\boldsymbol{x}}_{s} \cdot \dot{\boldsymbol{x}}_{s}+k \boldsymbol{x}_{s} \cdot \dot{\boldsymbol{x}}_{s}\right)=0 .
\end{aligned}
$$

Using Reynolds transport theorem, the first line of Eq. (34) can also be ex-$$
\int_{C V} \frac{D}{D t}\left(\frac{1}{2} \rho \boldsymbol{u} \cdot \boldsymbol{u}\right) d V=\frac{D}{D t} \int_{C V} \frac{1}{2} \rho \boldsymbol{u} \cdot \boldsymbol{u} d V+\int_{C S}\left(\frac{1}{2} \rho \boldsymbol{u} \cdot \boldsymbol{u}\right)\left(\boldsymbol{u}-\boldsymbol{v}_{C S}\right) \cdot \boldsymbol{n} d A
$$

No boundary condition has yet been applied in deriving Eq. (34). In the following section, the no-slip condition is included implicitly. 


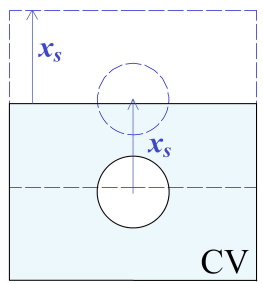

Figure 3: The selected control volume where the control volume moves rigidly with the structure. $\boldsymbol{x}_{s}$ denotes the displacement of the structure.

482

8.1. Reduced-Order Model with Implicit Implementation of the No-Slip Condition

The applicability of an analytical formulation is greatly affected by the selection of the control volume. For reasons that will become apparent, we consider the more restricted form of the control volume shown in Fig. 3, where the control volume moves rigidly with the structure. That is, the velocity of the structure equals the velocity of the control surface, $\dot{\boldsymbol{x}}_{s}=\boldsymbol{v}_{C S}$.

In Section 5 we discussed that, by excluding the dissipative terms, the remaining viscous terms together with the pressure represent the external loads on the closed surface and they can be viewed as conservative action and reaction forces where the no-slip condition is considered. Therefore, going through the same steps shown there, Eq. (34) becomes

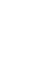

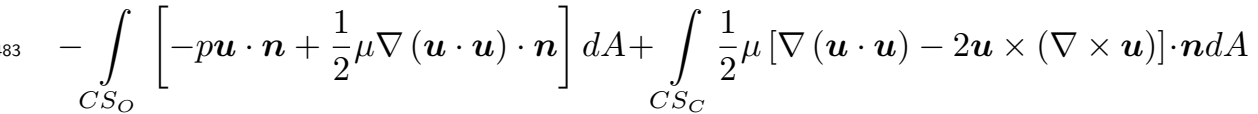

84

$$
\left.+\frac{1}{2} \int_{C V} \mu(\nabla \times \boldsymbol{u}) \cdot(\nabla \times \boldsymbol{u}) d V+\int_{C V} \nabla \cdot\left[\frac{1}{2} \rho(\boldsymbol{u} \cdot \boldsymbol{u}) \boldsymbol{u}\right] d V\right\}
$$$$
+\delta\left(m_{s} \ddot{\boldsymbol{x}}_{s} \cdot \dot{\boldsymbol{x}}_{s}+\frac{1}{2} C \dot{\boldsymbol{x}}_{s} \cdot \dot{\boldsymbol{x}}_{s}+k \boldsymbol{x}_{s} \cdot \dot{\boldsymbol{x}}_{s}\right)=0
$$

where the term $\left(\frac{1}{2} \rho \boldsymbol{u} \cdot \boldsymbol{u}\right)\left(\boldsymbol{u}-\dot{\boldsymbol{x}}_{s}\right)$ is taken inside the control volume, assuming that the fluid particles can shed from the structure. Thus, the no-slip condition 
is implemented by neglecting the viscous and pressure forces at the solid structure in Eq. (36). The no-penetration condition will be satisfied by integration over the appropriate control volume.

Having obtained the variational energy rate equation, we wish to obtain two coupled governing equations via the methodology explained in Section 7 . We start by applying Eq. (33) to Eq. (36) in order to obtain the rate of generalized momentum associated with $\boldsymbol{u}$. The terms not containing the flow velocity $\boldsymbol{u}$ will drop out and the terms expressed purely by $\boldsymbol{u}$ remain the same. Moreover, the terms of both velocities $\boldsymbol{u}$ and $\dot{\boldsymbol{x}}_{s}$ remain the same. Therefore, $\delta \dot{L}_{\boldsymbol{u}}$ is found to be

498

$\delta \dot{L}_{\boldsymbol{u}}=\delta\left\{\frac{D}{D t} \int_{C V} \frac{1}{2} \rho \boldsymbol{u} \cdot \boldsymbol{u} d V+\int_{C V} \nabla \cdot\left[\left(\frac{1}{2} \rho \boldsymbol{u} \cdot \boldsymbol{u}\right)\left(\boldsymbol{u}-\dot{\boldsymbol{x}}_{s}\right)\right] d V\right.$

$-\int_{C S_{O}}\left[-p \boldsymbol{u} \cdot \boldsymbol{n}+\frac{1}{2} \mu \nabla(\boldsymbol{u} \cdot \boldsymbol{u}) \cdot \boldsymbol{n}\right] d A+\int_{C S_{C}} \frac{1}{2} \mu[\nabla(\boldsymbol{u} \cdot \boldsymbol{u})-2 \boldsymbol{u} \times(\nabla \times \boldsymbol{u})] \cdot \boldsymbol{n} d A$

$\left.+\frac{1}{2} \int_{C V} \mu(\nabla \times \boldsymbol{u}) \cdot(\nabla \times \boldsymbol{u}) d V+\int_{C V} \nabla \cdot\left[\frac{1}{2} \rho(\boldsymbol{u} \cdot \boldsymbol{u}) \boldsymbol{u}\right] d V\right\}$.

The equation representing the participation of the fluid dynamic parameters is obtained by setting $\dot{L}_{\boldsymbol{u}}=0$.

For the structural terms, we apply Eqs. 28) and 29) (for $\dot{\boldsymbol{x}}_{s}$ instead of $\boldsymbol{u}$ ) to the remaining terms of the energy rate equation (Eq. (36)), which are

$$
\begin{aligned}
\delta \int_{C V} \nabla \cdot\left[\left(\frac{1}{2} \rho \boldsymbol{u} \cdot \boldsymbol{u}\right)\left(-\dot{\boldsymbol{x}}_{s}\right)\right] & d V+ \\
& \delta\left(m_{s} \ddot{\boldsymbol{x}}_{s} \cdot \dot{\boldsymbol{x}}_{s}+\frac{1}{2} C \dot{\boldsymbol{x}}_{s} \cdot \dot{\boldsymbol{x}}_{s}+k \boldsymbol{x}_{s} \cdot \dot{\boldsymbol{x}}_{s}\right)=0 .
\end{aligned}
$$

Considering the purely structural terms, we have

$$
\begin{aligned}
510 \quad \delta\left(m_{s} \ddot{\boldsymbol{x}}_{s} \cdot \dot{\boldsymbol{x}}_{s}+\frac{1}{2} C \dot{\boldsymbol{x}}_{s} \cdot \dot{\boldsymbol{x}}_{s}+k \boldsymbol{x}_{s} \cdot \dot{\boldsymbol{x}}_{s}\right) & =\left(m_{s} \ddot{\boldsymbol{x}}_{s}+C \dot{\boldsymbol{x}}_{s}+k \boldsymbol{x}_{s}\right) \cdot \delta \dot{\boldsymbol{x}}_{s} \\
& =\left(m_{s} \ddot{x}_{s}+C \dot{x}_{s}+k x_{s}\right) \delta \dot{x}_{s} .
\end{aligned}
$$

Manipulating the flux of the kinetic energy term using Gauss' theorem, we 
obtain

514

$$
\begin{aligned}
\delta \int_{C V} \nabla \cdot\left[\left(\frac{1}{2} \rho \boldsymbol{u} \cdot \boldsymbol{u}\right) \dot{\boldsymbol{x}}_{s}\right] d V & =\int_{C S}\left(\frac{1}{2} \rho \boldsymbol{u} \cdot \boldsymbol{u}\right)\left(\delta \dot{\boldsymbol{x}}_{s} \cdot \boldsymbol{n}\right) d A \\
& =\delta \dot{x}_{s}\left\{\int_{C V} \nabla\left(\frac{1}{2} \rho \boldsymbol{u} \cdot \boldsymbol{u}\right) \cdot \mathbf{e}_{x} d V\right\},
\end{aligned}
$$

where $\mathbf{e}_{x}$ is the unit vector in direction of $\dot{\boldsymbol{x}}_{s}$. Substituting Eqs. (39) and 40 into Eq. (38), and noting that $\delta \dot{x}_{s}$ is arbitrary, the structural EOM is found to be

$$
m_{s} \ddot{x}_{s}+C \dot{x}_{s}+k x_{s}=\int_{C V} \nabla\left(\frac{1}{2} \rho \boldsymbol{u} \cdot \boldsymbol{u}\right) \cdot \mathbf{e}_{x} d V .
$$

Finally, the general coupled equations of motion are obtained from Eqs. 37 and (41) to be

$$
\left\{\begin{array}{c}
m_{s} \ddot{x}_{s}+C \dot{x}_{s}+k x_{s}=\int_{C V} \nabla\left(\frac{1}{2} \rho \boldsymbol{u} \cdot \boldsymbol{u}\right) \cdot \mathbf{e}_{x} d V \\
\frac{D}{D t} \int_{C V} \frac{1}{2} \rho \boldsymbol{u} \cdot \boldsymbol{u} d V+\int_{C V} \nabla\left(\frac{1}{2} \rho \boldsymbol{u} \cdot \boldsymbol{u}\right) \cdot \boldsymbol{u} d V \\
\quad-\int_{C S_{O}}\left[-p \boldsymbol{u} \cdot \boldsymbol{n}+\frac{1}{2} \mu \nabla(\boldsymbol{u} \cdot \boldsymbol{u}) \cdot \boldsymbol{n}\right] d A \\
\quad+\int_{C S_{C}} \frac{1}{2} \mu[\nabla(\boldsymbol{u} \cdot \boldsymbol{u})-2 \boldsymbol{u} \times(\nabla \times \boldsymbol{u})] \cdot \boldsymbol{n} d A \\
+\int_{C V} \frac{1}{2} \mu(\nabla \times \boldsymbol{u}) \cdot(\nabla \times \boldsymbol{u}) d V+\int_{C V} \nabla\left[\frac{1}{2} \rho(\boldsymbol{u} \cdot \boldsymbol{u})\right] \cdot \boldsymbol{u} d V \\
=\int_{C V} \nabla\left(\frac{1}{2} \rho \boldsymbol{u} \cdot \boldsymbol{u}\right) \cdot \dot{\boldsymbol{x}}_{s} d V .
\end{array}\right.
$$

Having obtained the coupled equations for the model problem, we next show that a different equation can be obtained by a different approach to implementing the boundary conditions.

8.2. Reduced-Order Model with Explicit Implementation of the No-Slip Condition

In the previous section, we obtained a reduced-order model by implementing the no-slip condition implicitly, that is, by assuming the fluid forces at the solid 
surface have an equal and opposite reactions. Therefore, they do not affect the total energy of a system (based on d'Alembert's principle). Alternatively, in this section, we show that a different approach can be used if we wish to keep the interacting forces at the solid surface. Similar to the previous section, we assume that the no-slip condition holds. (Note that Dong et al. [5] did not consider any boundary conditions and yet they obtained a control volume where the predictions of their analytical model matched the experimental observation with very good accuracy.) We choose the control volume shown in Fig. 2 The open portion of control volume can contain closed surfaces and it may be selected to have shapes other than the rectangle shown in that figure.

We wish to introduce the no-slip condition into Eq. (35). Since the virtual velocities must be compatible with the system constraints and the no-slip condition is simply a nonholonomic constraint, the relation $\delta \dot{\boldsymbol{x}}_{s}-\delta \boldsymbol{u}=0$ must hold at the solid surface. Therefore, from the derivations of Section 6 and by dimensional considerations, we choose a no-slip condition of the form

$$
\delta \int_{C S_{C}}\left(\frac{1}{2} \rho \boldsymbol{u} \cdot \boldsymbol{u}\right)\left(\dot{\boldsymbol{x}}_{s}-\boldsymbol{u}\right) \cdot \boldsymbol{n} d A=0 .
$$


55

Since Eq. 43 equals zero for all time, we can add it to Eq. (34) to obtain

$$
\begin{aligned}
& \delta\{\int_{C V} \frac{D}{D t}\left(\frac{1}{2} \rho \boldsymbol{u} \cdot \boldsymbol{u}\right) d V+\overbrace{\int_{C S_{C}}\left(\frac{1}{2} \rho \boldsymbol{u} \cdot \boldsymbol{u}\right)\left(\dot{\boldsymbol{x}}_{s}-\boldsymbol{u}\right) \cdot \boldsymbol{n} d A}^{\text {no-slip condition }} \\
& \overbrace{-\int_{C S}\{-p \boldsymbol{u} \cdot \boldsymbol{n}+\mu[\nabla(\boldsymbol{u} \cdot \boldsymbol{u})-\boldsymbol{u} \times(\nabla \times \boldsymbol{u})] \cdot \boldsymbol{n}}^{\text {loading external to the fluid }}
\end{aligned}
$$

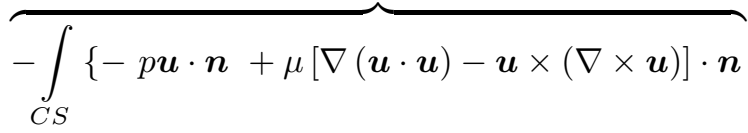

$$
\overbrace{\left.-\frac{1}{2} \mu[\nabla(\boldsymbol{u} \cdot \boldsymbol{u})-2 \boldsymbol{u} \times(\nabla \times \boldsymbol{u})] \cdot \boldsymbol{n}\right\} d A+\int_{C V} \frac{1}{2} \mu(\nabla \times \boldsymbol{u}) \cdot(\nabla \times \boldsymbol{u}) d V}^{\text {viscous dissipation }}
$$

$$
\begin{aligned}
& +\overbrace{\int_{C V} \nabla \cdot\left[\frac{1}{2} \rho(\boldsymbol{u} \cdot \boldsymbol{u}) \boldsymbol{u}\right] d V}^{\text {non-commuting term }}\} \\
& +\delta\left(m_{s} \ddot{\boldsymbol{x}}_{s} \cdot \dot{\boldsymbol{x}}_{s}+\frac{1}{2} C \dot{\boldsymbol{x}}_{s} \cdot \dot{\boldsymbol{x}}_{s}+k \boldsymbol{x}_{s} \cdot \dot{\boldsymbol{x}}_{s}\right)=0,
\end{aligned}
$$

where Eq. (35) is applied to the first line of Eq. (34). Summation of the viscous terms and expansion of the second and last integrals of Eq. (44) yields for the problem at hand

56

$$
\delta\left\{\int_{C V} \rho \frac{D}{D t}\left(\frac{1}{2} u^{2}\right) d V+\int_{C S_{C}} \rho\left(\frac{1}{2} u^{2}\right)\left(\dot{\boldsymbol{x}}_{s} \cdot \boldsymbol{n}\right) d A-\int_{C S_{C}} \rho\left(\frac{1}{2} u^{2}\right)(\boldsymbol{u} \cdot \boldsymbol{n}) d A\right.
$$$$
-\int_{C S}\left[-p \boldsymbol{u} \cdot \boldsymbol{n}+\mu \nabla\left(\frac{1}{2} u^{2}\right) \cdot \boldsymbol{n}\right] d A+\int_{C V} \frac{1}{2} \mu(\nabla \times \boldsymbol{u}) \cdot(\nabla \times \boldsymbol{u}) d V
$$$$
\left.+\int_{C S_{C}} \rho\left(\frac{1}{2} u^{2}\right)(\boldsymbol{u} \cdot \boldsymbol{n}) d A+\int_{C S_{O}} \rho\left(\frac{1}{2} u^{2}\right)(\boldsymbol{u} \cdot \boldsymbol{n}) d A\right\}
$$$$
+\delta\left(m_{s} \ddot{\boldsymbol{x}}_{s} \cdot \dot{\boldsymbol{x}}_{s}+\frac{1}{2} C \dot{\boldsymbol{x}}_{s} \cdot \dot{\boldsymbol{x}}_{s}+k \boldsymbol{x}_{s} \cdot \dot{\boldsymbol{x}}_{s}\right)=0 .
$$

Summing terms of the form $\int_{C S_{C}} \rho\left(\frac{1}{2} u^{2}\right)(\boldsymbol{u} \cdot \boldsymbol{n}) d A$ and applying Gauss' theorem to the viscous terms in the integrand of the fourth integral,

$$
\int_{C S} \frac{1}{2} \mu \nabla\left(u^{2}\right) \cdot \boldsymbol{n} d A=\int_{C V} \mu \nabla^{2}\left(\frac{1}{2} u^{2}\right) d V
$$


we obtain,

561

562

563

$$
\begin{gathered}
\delta\left\{\int_{C V} \rho \frac{D}{D t}\left(\frac{1}{2} u^{2}\right) d V+\int_{C S_{C}} \rho\left(\frac{1}{2} u^{2}\right)\left(\dot{\boldsymbol{x}}_{s} \cdot \boldsymbol{n}\right) d A+\int_{C S_{O}} \rho\left(\frac{1}{2} u^{2}\right)(\boldsymbol{u} \cdot \boldsymbol{n}) d A\right. \\
+\int_{C V}\left[\nabla p \cdot \boldsymbol{u}-\mu \nabla^{2}\left(\frac{1}{2} u^{2}\right) \cdot \boldsymbol{n}+\frac{1}{2} \mu(\nabla \times \boldsymbol{u}) \cdot(\nabla \times \boldsymbol{u})\right] d V \\
\left.+m_{s} \ddot{\boldsymbol{x}}_{s} \cdot \dot{\boldsymbol{x}}_{s}+\frac{1}{2} C \dot{\boldsymbol{x}}_{s} \cdot \dot{\boldsymbol{x}}_{s}+k \boldsymbol{x}_{s} \cdot \dot{\boldsymbol{x}}_{s}\right\}=0 .
\end{gathered}
$$

Setting $\dot{L}_{\boldsymbol{u}}=0$ and $\partial \dot{L} / \partial \dot{\boldsymbol{x}}_{s}=0$, results in

$$
\left\{\begin{array}{c}
m_{s} \ddot{x}_{s}+C \dot{x}_{s}+k x_{s}=-\int_{C S_{C}} \rho\left(\frac{1}{2} u^{2}\right)\left(\mathbf{e}_{x} \cdot \boldsymbol{n}\right) d A, \\
\int_{C V} \rho \frac{D}{D t}\left(\frac{1}{2} u^{2}\right) d V+\int_{C S_{O}} \rho\left(\frac{1}{2} u^{2}\right)(\boldsymbol{u} \cdot \boldsymbol{n}) d A \\
+\int_{C V}\left[\nabla p \cdot \boldsymbol{u}-\mu \nabla^{2}\left(\frac{1}{2} u^{2}\right)+\frac{1}{2} \mu(\nabla \times \boldsymbol{u}) \cdot(\nabla \times \boldsymbol{u})\right] d V \\
=-\dot{x}_{s} \int_{C S_{C}} \rho\left(\frac{1}{2} u^{2}\right)\left(\mathbf{e}_{x} \cdot \boldsymbol{n}\right) d A,
\end{array}\right.
$$

where $u^{2}=\boldsymbol{u} \cdot \boldsymbol{u}$ and the required steps are similar to those of Section 8.1.

Thus far, we have obtained two possible reduced-order sets of governing equations for the model problem in the form of a translating cylinder. Similar reduced-order models for the inverted pendulum problem can be easily obtained and can be found in $[12$.

As evident from our derivations thus far, depending on our interpretation of the boundary conditions and on how we choose to implement them, the resulting reduced order model differs to some extent. Both Eqs. 42 and (48), or any other models obtained by implementing the boundary conditions differently than those shown, can be simplified further by dimensional analysis, similarity methods or perturbation techniques. However, experimental data and observations are required for further simplifications.

In order to show the steps required, as an example, we consider the experimental observations of Benaroya and Wei [10] and Dong et al. [5] to obtain a 
model similar to the wake oscillator proposed by Hartlen and Currie. To accomplish this, we will not use similarity methods in a traditional way. However, we will make a few assumptions based on their experimental observations. We emphasize that we do this only to demonstrate that the equations we derived above can be interpreted to embody the flow-oscillators published in the literature. To demonstrate this requires specialization of our equations but does not imply limited applicability of those equations.

\section{Modeling VIV: A Lift-Oscillator Model}

Our main goal in this research work has been to obtain first-principle based equations governing vortex-induced structural oscillations, which we accomplished in the previous sections with Eqs. (42) and (48). The results are two coupled governing equations, one a differential equation representing the rigid structure and the other an integral equation representing the coupled fluid dynamics.

However, wake-oscillator models in the literature are generally two coupled differential equations, one linear and one nonlinear. In order to show that such equations can be obtained from, for example, Eq. (48), we seek a reduced order model similar to the lift-oscillator model of Hartlen and Currie [4]. Their model has been chosen since it is one of the earliest and, perhaps, the most noteworthy of the wake-oscillator models.

We start our manipulations with some general assumptions that can be made for many VIV problems and apply these to the reduced-order model Eq. 48.

For fluids with very low viscosity, the boundary layer approximation method, proposed by Prandtl, assumes that viscosity only exists in the vicinity of the solid structure, and is neglected elsewhere. Following Prandtl's hypothesis, we neglect the viscous dissipation inside the control volume, $\int_{C V} \frac{1}{2} \mu(\nabla \times \boldsymbol{u})$. 
$(\nabla \times \boldsymbol{u}) d V \approx 0,($ see Eq. (24) $)$ in Eq. [48), resulting in

$$
\left\{\begin{aligned}
& m_{s} \ddot{x}_{s}+C \dot{x}_{s}+k x_{s}=-\int_{C S_{C}} \rho \hat{T}\left(\mathbf{e}_{x} \cdot \boldsymbol{n}\right) d A \\
& \int_{C V} \rho \frac{D \hat{T}}{D t} d V+\int_{C S_{O}} \rho \hat{T}(\boldsymbol{u} \cdot \boldsymbol{n}) d A+\int_{C V}\left(\nabla p \cdot \boldsymbol{u}-\mu \nabla^{2} \hat{T}\right) d V \\
&=-\dot{x}_{s} \int_{C S_{C}} \rho \hat{T}\left(\mathbf{e}_{x} \cdot \boldsymbol{n}\right) d A,
\end{aligned}\right.
$$

where the kinetic energy density function is $\hat{T}=u^{2} / 2$. In order to simplify Eq. 49 further, we consider some experimental observations of Dong et al. [5].

Dong et al. performed a series of experiments on the VIV model problem in the form of an inverted pendulum, while we have considered the model problem in the form of a translating cylinder. However, due to similarities between the two problems, we assume similar results would be found for the problem at hand. Moreover, the control volume of their experiments was selected such that the downstream control surface views the structured vortices, that is, it was close enough to the structure so that the shed vortices had not yet broken down into smaller eddies.

Since Dong et al. considered the rate of kinetic energy to be $\int_{C V} \frac{\partial \rho \hat{T}}{\partial t} d V$, we extract the same integral from the first integral in the second equation of Eq. (49) and obtain

$620 \quad\left\{\begin{array}{l}m_{s} \ddot{x}_{s}+C \dot{x}_{s}+k x_{s}=-\int_{C S_{C}} \rho \hat{T}\left(\mathbf{e}_{x} \cdot \boldsymbol{n}\right) d A, \\ \int_{C V} \rho \frac{\partial \hat{T}}{\partial t} d V+\int_{C S_{C}} \rho \hat{T}(\boldsymbol{u} \cdot \boldsymbol{n}) d A+2 \int_{C S_{O}} \rho \hat{T}(\boldsymbol{u} \cdot \boldsymbol{n}) d A \\ +\int_{C V}\left(\nabla p \cdot \boldsymbol{u}-\mu \nabla^{2} \hat{T}\right) d V=-\dot{x}_{s} \int_{C S_{C}} \rho \hat{T}\left(\mathbf{e}_{x} \cdot \boldsymbol{n}\right) d A .\end{array}\right.$

Next we interpret the experimental observations of Dong et al. so that they become applicable to Eq. (50).

Based on the observation by Dong et al. that the flux term at the closed 
surface corresponds to the structural oscillation, we assume the relation

$$
\int_{C S_{C}} \rho \hat{T}(\boldsymbol{x}, t)[\boldsymbol{u}(\boldsymbol{x}, t) \cdot \boldsymbol{n}] d A=\int_{C S_{C}} \rho \hat{T}\left(\boldsymbol{x}, t+\frac{1}{f_{s}}\right)\left[\boldsymbol{u}\left(\boldsymbol{x}, t+\frac{1}{f_{s}}\right) \cdot \boldsymbol{n}\right] d A,
$$

where $f_{s}(\mathrm{~Hz})$ is the frequency of structural oscillation. Eq. (51) represents a boundary condition and since we have two coupled equations it must only be applied to one of them. Since the no-slip condition is a boundary condition for the fluid and not the structure, we make the substitution in the governing equation of the fluid (second equation) in Eq. (50). Moreover, the terms $\int_{C S_{C}} \rho \hat{T}(\boldsymbol{u} \cdot \boldsymbol{n}) d A$ and $\dot{x}_{s} \int_{C S_{C}} \rho \hat{T}\left(\mathbf{e}_{x} \cdot \boldsymbol{n}\right) d A$ vary with the same frequency, thus we assume that their superposition also varies with the same frequency. Therefore,

$$
\alpha \dot{x}_{s} \cong \dot{x}_{s} \int_{C S_{C}} \rho \hat{T}\left(\mathbf{e}_{x} \cdot \boldsymbol{n}\right) d A+\int_{C S_{C}} \rho \hat{T}(\boldsymbol{u} \cdot \boldsymbol{n}) d A
$$

where $\alpha$ is a constant with dimension of force and the integrals are evaluated from the experimental measurements in the vicinity of the structure. This relaxes the condition on the corresponding term in the structural differential equation. In Eq. (52),

$$
\int_{C S_{C}} \rho \hat{T}(\boldsymbol{x}, t)\left(\mathbf{e}_{x} \cdot \boldsymbol{n}\right) d A \equiv \int_{C S_{C}} \rho \hat{T}\left(\boldsymbol{x}, t+\frac{1}{f_{v}}\right)\left(\mathbf{e}_{x} \cdot \boldsymbol{n}\right) d A,
$$

where $f_{v}(\mathrm{~Hz})$ is the frequency of vortex shedding.

Considering the second equation in Eq. (50) and introducing Eq. 52, we have

$$
\int_{C V} \rho \frac{\partial \hat{T}}{\partial t} d V+2 \int_{C S_{O}} \rho \hat{T}(\boldsymbol{u} \cdot \boldsymbol{n}) d A+\int_{C V}\left(\nabla p \cdot \boldsymbol{u}-\mu \nabla^{2} \hat{T}\right) d V=-\alpha \dot{x}_{s} .
$$

40 Dong et al. found that the time rate of change of the kinetic energy of the system was correlated with the vortex shedding frequency. Therefore,

$$
\int_{C V} \rho \frac{\partial}{\partial t} \hat{T}(\boldsymbol{x}, t) d V=\int_{C V} \rho \frac{\partial}{\partial t} \hat{T}\left(\boldsymbol{x}, t+\frac{1}{f_{v}}\right) d V .
$$

At this point, we choose the direction of free-stream flow to be the $z$ axis of the Cartesian coordinate system defined in Fig. 4. Therefore, $\hat{T}(\boldsymbol{x}, t)=$ 


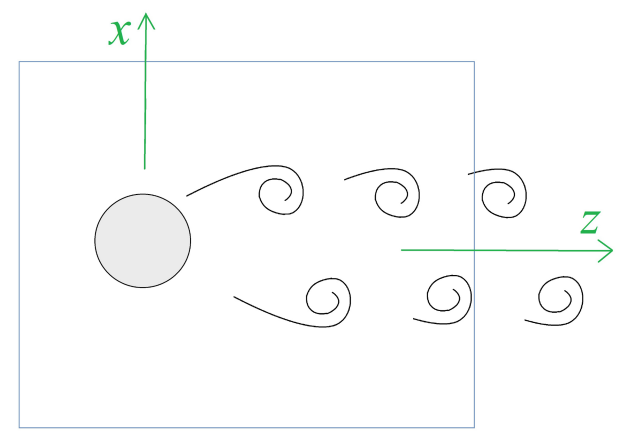

Figure 4: A schematic diagram of the experiments of Dong et al. where the shedded vortices are $P+S$ type vortices. For definition of vortices names one may refer to [2].

$\hat{T}(x, z, t)$. Also, both in experimental and analytical studies, the flow is controlled or considered to be two dimensional for all time, as are the shedding vortices [2], because the coherent vortices result in the largest structural oscillation (the worst-case scenario). That is, $u_{y}=0$.

Since no external or dissipative forces are present inside the control volume (due to Prandtl hypothesis), a shed vortex must repeat its pattern periodically as it travels in the direction of the free-stream flow. Therefore, we have

$$
\hat{T}(x, z, t) d=\hat{T}\left(x, z-c_{v} t, t\right),
$$

where $c_{v}$ is the velocity of shed vortices.

Considering Eqs. 55$)$ and $(56, \hat{T}(\boldsymbol{x}, t)$ has the characteristics of a propagating wave with velocity $c_{v}$ and frequency $f_{v}$. Therefore, from the wave equation, we have

$$
\frac{\partial^{2} \hat{T}(\boldsymbol{x}, t)}{\partial t^{2}} \equiv c_{v}^{2} \nabla^{2} \hat{T}(\boldsymbol{x}, t) .
$$

Introducing Eq. (57) into Eq. 54, yields

$$
\int_{C V} \rho \frac{\partial \hat{T}}{\partial t} d V+2 \int_{C S_{O}} \rho \hat{T}(\boldsymbol{u} \cdot \boldsymbol{n}) d A+\int_{C V} \nabla p \cdot \boldsymbol{u} d V-\int_{C V} \frac{\mu}{c_{v}^{2}} \frac{\partial^{2} \hat{T}(\boldsymbol{x}, t)}{\partial t^{2}} d V=-\alpha \dot{x}_{s} .
$$

Based on the experiments of Dong et al., the flux of the kinetic energy across the open control volume was found to vary with the frequency of the vortex 
shedding, that is,

$$
\int_{C S_{O}} \rho \hat{T}(\boldsymbol{x}, t)[\boldsymbol{u}(\boldsymbol{x}, t) \cdot \boldsymbol{n}] d A=\int_{C S_{O}} \rho \hat{T}\left(\boldsymbol{x}, t+\frac{1}{f_{v}}\right)\left[\boldsymbol{u}\left(\boldsymbol{x}, t+\frac{1}{f_{v}}\right) \cdot \boldsymbol{n}\right] d A .
$$

However, the kinetic energy inside the control volume must also vary with the same frequency when Eq. (55) is considered. Moreover, the flux of the kinetic energy adds and subtracts energy from the system very similarly to a spring. Since, $\hat{T}(\boldsymbol{x}, t)$ and $\hat{T}(\boldsymbol{x}, t) \boldsymbol{u}(\boldsymbol{x}, t)$ are harmonic functions with frequencies that are multiples of the shedding frequency, and since the solution of the velocity field inside the control volume is a function of the boundary conditions, we can write

$$
\int_{C S_{O}} \rho \hat{T}(\boldsymbol{x}, t)[\boldsymbol{u}(\boldsymbol{x}, t) \cdot \boldsymbol{n}] d A_{\sim}^{\propto}-\int_{C V} \rho f_{v} \hat{T}(\boldsymbol{x}, t) d V,
$$

where $\underset{\sim}{\sim}$ means they are approximately proportional and the negative sign is introduced to show that the right-hand side is positive when the left-hand side is negative. By introducing the constant $\beta$, we have the equality

$$
\int_{C S_{O}} \rho \hat{T}(\boldsymbol{x}, t)[\boldsymbol{u}(\boldsymbol{x}, t) \cdot \boldsymbol{n}] d A=-\int_{C V} \beta \rho f_{v} \hat{T}(\boldsymbol{x}, t) d V,
$$

where $\beta$ is to be determined. Substituting Eq. (61) into Eq. (58), we obtain

${ }_{669}-\frac{\mu}{c_{v}^{2}} \int_{C V} \frac{\partial^{2} \hat{T}(\boldsymbol{x}, t)}{\partial t^{2}} d V+\int_{C V} \rho \frac{\partial \hat{T}}{\partial t} d V-\int_{C V} 2 \beta f_{v} \rho \hat{T} d V+\int_{C V} \nabla p \cdot \boldsymbol{u} d V=-\alpha \dot{x}_{s}$.

From Gauss' divergence theorem we expect that the term $\int_{C S_{C}} \rho \hat{T}\left(\mathbf{e}_{x} \cdot \boldsymbol{n}\right) d A$ in Eq. 50 be approximately proportional to $\int_{C V} \nabla(\rho \hat{T}) \cdot \mathbf{e}_{x} d V$. As mentioned earlier, $\hat{T}(\boldsymbol{x}, t)$ has the characteristics of a propagating wave,

$$
\frac{\partial \hat{T}(\boldsymbol{x}, t)}{\partial t} \equiv-c_{v} \nabla \hat{T}(\boldsymbol{x}, t)
$$

and therefore, we assume equality and obtain

$$
\int_{C S_{C}} \rho \hat{T}\left(\mathbf{e}_{x} \cdot \boldsymbol{n}\right) d A=-\int_{C V} \gamma \frac{1}{c_{v}} \rho \frac{\partial \hat{T}(\boldsymbol{x}, t)}{\partial t} d V,
$$


where $\gamma$ is a constant to be determined. It is important to note that in Eqs. 61 and (64), constants $\beta$ and $\gamma$ can more generally be replaced with functions of $f_{v}$ and $f_{v} / c_{v}$, respectively, if the vortices are not structured in a velocity range of interest. However, the vortices were fairly structured at the flow velocity examined in [5, 10].

Substituting Eqs. (62) and (64) into Eq. (50) yields

$$
\left\{\begin{array}{l}
m_{s} \ddot{x}_{s}+C \dot{x}_{s}+k x_{s}=\int_{C V} \gamma \frac{1}{c_{v}} \rho \frac{\partial \hat{T}(\boldsymbol{x}, t)}{\partial t} d V \\
-\frac{\mu}{c_{v}^{2}} \int_{C V} \frac{\partial^{2} \hat{T}(\boldsymbol{x}, t)}{\partial t^{2}} d V+\int_{C V} \rho \frac{\partial \hat{T}}{\partial t} d V-\int_{C V} 2 \beta f_{v} \rho \hat{T} d V+\int_{C V} \nabla p \cdot \boldsymbol{u} d V=-\alpha \dot{x}_{s} .
\end{array}\right.
$$

Thus far, we have not considered the pressure terms, since they are perhaps the most complex and challenging terms. The pressure is the thermodynamic pressure and it is assumed to be independent of velocity in the derivation of the constitutive relation for Newtonian incompressible fluids. Yet, its distribution is often found to be a function of velocity. For example, for the problems with steady low speed flow, the pressure distribution is found via Bernoulli's equation which leads to defining a nondimensional excess pressure, called the pressure coefficient $C_{p}$,

$$
C_{p} \equiv \frac{p-p_{\infty}}{\frac{1}{2} \rho u_{\infty}^{2}},
$$

where $p_{\infty}$ and $u_{\infty}$ are the pressure and velocity at infinity.

While the pressure coefficient was originally defined for steady flow, it has been used extensively in similarity analysis of complicated non-linear problems by assuming $C_{p}$ to be a function of nondimentional fluid parameters [15].

The experiments of Dong et al. found that the work done by the pressure was correlated with the rate of kinetic energy, that is,

$$
p(\boldsymbol{x}, t)=p\left(\boldsymbol{x}, t+\frac{1}{f_{v}}\right) .
$$

Based on Eq. (66), we expect a relation between pressure and kinetic energy of the form

$$
p(\boldsymbol{x}, t)=f[\rho \hat{T}(\boldsymbol{x}, t)],
$$


where $f$ is a function to be determined. Furthermore, since $\hat{T}(\boldsymbol{x}, t)$ behaves similarly to a wave, that is,

$$
\begin{aligned}
p(\boldsymbol{x}, t) & =f[\rho \hat{T}(\boldsymbol{x}, t)] \\
& =f\left[\rho \hat{T}\left(\boldsymbol{x}, t+\frac{1}{f_{v}}\right)\right] \\
& =f\left[\rho \hat{T}\left(\boldsymbol{x}-c_{v} t, t\right)\right],
\end{aligned}
$$

$p$ itself must be in the form of a wave. Therefore, $\partial p / \partial t \equiv-c_{v} \nabla p$. Consequently, we can say $\nabla p(\boldsymbol{x}, t)$ must be a function of $\frac{\partial}{\partial t} \hat{T}(\boldsymbol{x}, t)$, that is,

$$
\nabla p(\boldsymbol{x}, t)=-\frac{1}{c_{v}} \boldsymbol{f}\left[\frac{\partial}{\partial t} \hat{T}(\boldsymbol{x}, t)\right],
$$

where $f$ is a vector-valued function to be determined. Substituting Eq. 70 into Eq. 65, we obtain

$$
704 \quad\left\{\begin{array}{r}
m_{s} \ddot{x}_{s}+C \dot{x}_{s}+k x_{s}=\frac{\gamma}{c_{v}} \int_{C V} \rho \frac{\partial \hat{T}(\boldsymbol{x}, t)}{\partial t} d V \\
\frac{\mu}{c_{v}^{2}} \int_{C V} \frac{\partial^{2} \hat{T}(\boldsymbol{x}, t)}{\partial t^{2}} d V-\int_{C V} \rho \frac{\partial \hat{T}}{\partial t} d V+\int_{C V} \frac{1}{c_{v}} \boldsymbol{f}\left[\frac{\partial \hat{T}(\boldsymbol{x}, t)}{\partial t}\right] \cdot \boldsymbol{u} d V \\
+\int_{C V} 2 \beta f_{v} \rho \hat{T} d V=\alpha \dot{x}_{s} .
\end{array}\right.
$$

Defining an instantaneous average kinetic energy function for the control volume of an incompressible fluid by

$$
T(t)=\int_{C V} \rho \hat{T} d V,
$$

and introducing it into Eq. (71), we obtain

$$
\left\{\begin{array}{l}
m_{s} \ddot{x}_{s}+C \dot{x}_{s}+k x_{s}=\frac{\gamma}{c_{v}} \dot{T} \\
\frac{\mu}{\rho c_{v}^{2}} \ddot{T}-\dot{T}+f(\dot{T})+2 \beta f_{v} T=\alpha \dot{x}_{s},
\end{array}\right.
$$

where $\alpha, \beta$ and $\gamma$ are defined by Eqs. 52, 61 and 64 , respectively; $f(\dot{T})$ is a function with an order higher than one with respect to $\dot{T}$, with a form of that must be selected based on experimental considerations. Since the sign of $f(\dot{T})$ must be positive, Eq. 73 is a van der Pol type oscillator. 
For comparison with Hartlen and Currie, we may assume that $f(\dot{T})$ is the first order term of the Taylor series of a sine function. We select

$$
f(\dot{T})=\frac{\eta}{\rho f_{v} c_{v}^{4}} \dot{T}^{3}
$$

where the negative sign is eliminated since $f(\dot{T})$ must be a positive-valued function, and $\eta$ is a constant found experimentally. Based on this choice, the reduced-order model becomes

$$
\left\{\begin{array}{l}
m_{s} \ddot{x}_{s}+C \dot{x}_{s}+k x_{s}=\frac{\gamma}{c_{v}} \dot{T} \\
\frac{\mu}{\rho c_{v}^{2}} \ddot{T}-\dot{T}+\frac{\eta}{\rho f_{v} c_{v}^{4}} \dot{T}^{3}+2 \beta f_{v} T=\alpha \dot{x}_{s} .
\end{array}\right.
$$

It worth noting that one may choose to keep the zeroth order term of the $f(\dot{T})$ due to experimental considerations. This will introduce another constant that must be evaluated together with $\eta$ from curve fitting to particular experimental measurements.

Thus far, we have obtained reduced order models in the form of single governing EOM and two coupled EOM. Next, a comparison is made between our derived equations and some of the existing models in the literature.

\section{Comparisons with Three Existing Models}

\subsection{Comparison with McIver's Extension of Hamilton's Principle}

McIver extended Hamilton's principle for systems involving fluid-structure interactions 8 , specifically systems where the fluid is internal to the structure. He presented his energy rate equation in the absence of any interacting system as

$$
\begin{aligned}
& \frac{d}{d t} \int_{C V} \frac{1}{2} \rho u^{2} d V \\
& \quad=\int_{C S}\left\{\boldsymbol{u} \cdot\left[-p \overline{\boldsymbol{I}}+\mu\left(\nabla \boldsymbol{u}+\nabla^{T} \boldsymbol{u}\right)\right]+\left(\frac{1}{2} \rho u^{2}\right)\left(\boldsymbol{v}_{C S}-\boldsymbol{u}\right)\right\} \cdot \boldsymbol{n} d A .
\end{aligned}
$$

where we neglected the potential energy terms as we did in our derivations. 
For comparison, we manipulate the terms of our energy rate equation for fluid systems, Eq. (8), to match those of McIver's equation, the energy rate equation becomes

(3)

$$
\begin{aligned}
& \frac{D}{D t} \int_{C V} \frac{1}{2} \rho u^{2} d V=\overbrace{2\left\{\int_{C S}-\frac{1}{2} \mu[\nabla(\boldsymbol{u} \cdot \boldsymbol{u})-\ldots] \cdot \boldsymbol{n} d A-\frac{1}{2} \int_{C V} \ldots d V\right\}}^{\text {viscous dissipation }} \\
& +\int_{C S}\left\{\boldsymbol{u} \cdot\left[-p \overline{\boldsymbol{I}}+\mu\left(\nabla \boldsymbol{u}+\nabla^{T} \boldsymbol{u}\right)\right]+\left(\frac{1}{2} \rho u^{2}\right)\left(\boldsymbol{v}_{C S}-\boldsymbol{u}\right)+\left(\frac{1}{2} \rho u^{2}\right) \boldsymbol{u}\right\} \cdot \boldsymbol{n} d A
\end{aligned}
$$

(3)

Comparing Eq. (77) with Eq. (76), two important differences become clear.

The first difference is due to McIver's assumption that the only virtual work applied to a control volume is due to the surface traction over the control surfaces. Therefore, McIver assumes that there is no dissipation of energy due to viscousity. The second difference is the additional power term due to the non-commuting part of the rate of kinetic energy, $\left(\frac{1}{2} \rho u^{2}\right) \boldsymbol{u}$ in our energy rate equation. This term was obtained after examining the commutation rule in the Eulerian reference frame in another of our papers [11, 12]. However, McIver assumed the commutation rule holds and he did not consider the relations between the Lagrangian and the Eulerian reference frames.

For an incompressible fluid, the thermodynamic pressure can be considered to be a conservative force. McIver considered the energy equation for a control volume of fluid particles in the absence of a solid system to be conserved if the control volume is selected such that the relation

$$
\boldsymbol{v}_{C S} \cdot \boldsymbol{n}=-\frac{\boldsymbol{u} \cdot \overline{\boldsymbol{\sigma}} \cdot \boldsymbol{n}}{\rho\left(\frac{1}{2} u^{2}+e\right)}+\boldsymbol{u} \cdot \boldsymbol{n}
$$

holds on the open portion of the control volume. In Eq. $78, \bar{\sigma}$ is the stress dyadic and $e$ represents the potential energy. For FSI and VIV, the selection of a control volume such that it satisfies this relation is very challenging. It might be easily recognizable for systems where an approximately laminar flow is internal to the structure, but it is ambiguous otherwise. Thus, it is very challenging 
to compare McIver's method with ours any further since McIver's appropriate control volume cannot be distinguished without knowing the velocity field of the flow.

Another interesting extension of Hamilton's principle was by Benaroya and Wei [10], which is considered in the following section.

\subsection{Comparison with Benaroya and Wei's Extension of Hamilton's Principle}

Benaroya and Wei discussed in detail that in the absence of known particle trajectories for the fluid flow, stationarity cannot be ensured. Thus, setting $\delta \boldsymbol{r}=\boldsymbol{u} d t$ would lead to the conservation of energy equation. Based on their experimental measurements, they neglected the viscous dissipation of energy inside the control volume and obtained the energy equation for the translating cylinder problem (Fig. 1a) to be,

$$
\begin{aligned}
& \dot{\boldsymbol{x}}_{s}\left(m_{s} \ddot{\boldsymbol{x}}_{s}+k \boldsymbol{x}_{s}\right)+\left(m_{\text {fluid }} u \dot{u}\right)_{C V}= \\
& \int_{C S} \frac{1}{2} \rho u^{2}\left(\boldsymbol{u}_{r} \cdot \boldsymbol{n}\right) d A+\int_{C S_{O}}(-p \boldsymbol{n}+\boldsymbol{\tau}) \cdot \boldsymbol{u} d A+\int_{C S_{C}}(-p \boldsymbol{n}+\boldsymbol{\tau}) \cdot \boldsymbol{u} d A,
\end{aligned}
$$

where they have ignored the structural damping.

Comparing Benaroya and Wei's result (Eq. (79) ) to ours (Eq. (25p), we notice that the sign of the flux of kinetic energy in our equation is different than theirs. The reason is that Benaroya and Wei used the RTT to relate the integration over the control system to that over the control volume while we used the RTT twice when deriving our energy equation in [11, 12]; once for the same purpose and once to relate the change of control volume to itself.

Moreover, Benaroya and Wei considered the pressure and shear forces at the closed surface which we neglect by considering them to be action and reaction forces that do not appear in the energy equation. Regarding these terms, their equation also differs from that obtained by McIver.

Additionally, we notice similar differences to those observed in Section 10.1 . that is, the dissipation terms and the non-commuting term of the rate of kinetic energy are not present in their formulation. 
Finally, we compare our lift-oscillator model with that proposed by Hartlen and Currie.

\subsection{Comparison with Hartlen and Currie's lift-oscillator model}

The lift-oscillator model proposed by Hartlen and Currie was not derived from a first-principles approach. In Section 9, we derived Eq. (75), a model similar to the lift-oscillator model. Comparison of this model to Hartlen and Currie's model, Eq. (1), reveals a few differences that are worth noting, as follows.

The first difference is that the constants of Hartlen and Currie's model $(\alpha$, $\beta$, and $\gamma$ ) are meant to be used to fit the assumed EOM to a particular data set. These constants do not correspond to any dynamical parameter of the fluid flow and therefore cannot provide any physical insight. Contrary to their model, the constants $\alpha, \beta, \gamma$ and $\eta$ of the model developed here are obtained from specific assumptions made regarding fluid dynamic behavior.

As mentioned earlier, only two of the unknown fluid parameters of Hartlen and Currie's model must be selected in order to obtain the best fit to an experimental data set [5]. However, in our model, only the constant $\eta$ is obtained by curve fitting, but its physical meaning is well understood from Eq. (74).

Another difference observed between the two models is how the two EOMs are coupled. To clarify this statement, let us assume that the nonlinear EOM representing the fluid flow is a second oscillator coupled with the structure. In Hartlen and Currie's model, the structural EOM observes the forcing function due to $c_{L}$, that is, the displacement of the flow oscillator. However, in our model the structural EOM is coupled to the flow oscillator via the velocity, $\dot{T}$. Hartlen and Currie's model cannot represent beating behavior [5], which can be envisioned to be a result of the superposition of two waves with different frequencies. If Hartlen and Currie's model does not permit beating behavior, then the two oscillators are in phase. Since velocity is out of phase with respect to displacement, then our coupling via $\dot{T}$ allows for a beating behavior. 


\section{Conclusions}

While the importance of fluid-structure interaction has been well understood for many years, there exist no compelling analytical methods where the reducedorder models are derived via first-principles. In the past few decades, the few attempts to utilize variational principles in modeling FSI systems have had minimal success. And these models were not directly obtained from variational operations and required ad hoc assumptions. To overcome this difficulty, we extended Jourdain's principle for the modeling of FSI systems for a Newtonian incompressible viscous fluid. As examples, we considered the model problem in the form of a translating cylinder and inverted pendulum (shown in Figs. 1a and $1 \mathrm{~b}$ and obtained two types of reduced order models in the form of a Single governing EOM (SEOM) and in the form of two coupled EOMs.

Regarding the SEOM model, we used Benaroya and Wei's methodology to extend our energy rate equation for such systems. This method requires the pressure to be known at the surface of the structure. PIV and DPIV methods only estimate the velocity field. Thus, the pressure term is problematic. To obtain the pressure, Benaroya and Wei integrated the Navier-Stokes equation numerically. In our derivations, we neglect the pressure terms by considering them to be action and reaction forces. However, the pressure remains a requirement for the open sections of the control surface.

Our method, based on the important concept of generalized momentum in analytical mechanics, uses the rate of generalized momenta to obtain reducedorder coupled governing equations from the variational energy rate equation (extended JP).

We obtained two coupled equations, one a differential equation and the other an integral equation. We showed the importance of boundary conditions and their implementation by obtaining two different possible reduced-order models for the model problem in the form of translating cylinder (Eqs. 42 and (48)).

The wake-oscillator models available in the literature are ad hoc models, obtained by guessing a function that can capture some characteristics of a system. 
Therefore, they do not provide any insights into the fluid dynamic parameters that can affect the outcome. Nevertheless, they are valuable for engineering applications. As an example, we considered one the most noteworthy of those models, that was proposed by Hartlen and Currie. Their lift-oscillator model captures many features of the structural response without addressing the fluid dynamics.

Referring to Eq. (73), the method developed in this paper has the advantage that the resulting reduced-order model is expressed in terms of the kinetic energy of the control volume. Each of the terms in Eq. 73 has a specific meaning that can be traced back to the energy equation, and even further back to the NavierStokes equation. There exist no reduced-order models in the literature that are obtained without an assumed lift or drag coefficient function. The reducedorder modeling method proposed here, in its general form, does not require any such ad hoc assumptions. The coupled equations are obtained directly from the energy equation by using a variational method based on first principles, but we recognize that the specific derivation of our actual flow oscillator equations required a prior knowledge of the particular physical problem. While there are assumptions at this stage of the work, these are chosen based on a reading of experimental data. The primary contribution of our work is with the general derivations surrounding Jourdain's principle and the formulation in Eulerian form, along with application to fluid-structure systems.

We expect that the method developed here can be extended to compressible flows and elastic structures.

\section{Acknowledgement}

The writers thank Dr. Tom Swean and the Office of Naval Research (ONR, Grant No. N00014-12-1-0016) for his long-term interest and support of this effort. 


\section{References}

[1] R. D. Gabbai, H. Benaroya, A first-principles derivation procedure for wake-body models in vortex-induced vibration: Proof-of-concept, Journal of Sound and Vibration 312 (2008) $19-38$.

[2] R. D. Gabbai, H. Benaroya, An overview of modeling and experiments of vortex-induced vibration of circular cylinders, Journal of Sound and Vibration 282 (2005) 575-616.

[3] T. Sarpkaya, A critical review of the intrinsic nature of vortex-induced vibrations, Journal of Fluids and Structures 19 (4) (2004) 389-447.

[4] R. T. Hartlen, I. G. Currie, Lift-oscillator model of vortex induced vibration, Journal of the Engineering Mechanics 96 (5) (1970) 577-591.

[5] P. Dong, H. Benaroya, T. Wei, Integrating experiments into an energybased reduced-order model for vortex-induced-vibrations of a cylinder mounted as an inverted pendulum, Journal of Sound and Vibration 276 (12) (2004) 45-63.

[6] R. D. Gabbai, Hamilton's Principle for Fluid-Structure Interaction and Applications to the Free-Vibration of an Elastically-Mounted Cylinder, Ph.D. dissertation, Rutgers, the State University of New Jersey, New Brunswick, NJ, 2006.

[7] H. Benaroya, R. D. Gabbai, Modelling vortex-induced fluid-structure interaction, Philosophical Transactions: Mathematical, Physical and Engineering Sciences 366 (1868) (2008) 1231-1274.

[8] D. B. McIver, Hamilton's principle for systems of changing mass, Journal of Engineering Mechanics 7 (3) (1973) 249-261.

[9] J. T. Xing, W. G. Price, The theory of non-linear elastic ship-water interaction dynamics, Journal of Sound and Vibration 230 (4) (2000) 877-914. 
[10] H. Benaroya, T. Wei, Hamilton's principle for external viscous fluidstructure interaction, Journal of Sound and Vibration 238 (2000) 113 145.

[11] S. Mottaghi, H. Benaroya, An extension of jourdain's principle as applicable to fluid and fluid-structure interaction systems., Under Preparation.

[12] S. Mottaghi, Modeling vortex-induced fluid-structure interaction using an extension of jourdain's principle, Ph.D. thesis, Rutgers, the State University of New Jersey, New Brunswick, NJ (January 2015).

[13] S. Goldstein, Fluid mechanics in the first half of this century., Annual Review of Fluid Mechanics 1 (1) (1969) 1-29.

[14] C. Tropea, A. L. Yarin, J. F. Foss (Eds.), Springer Handbook of Experimental Fluid Mechanics., Vol. 1, Springer, 2007.

[15] P. Kundu, I. Cohen, Fluid Mechanics, 4th Edition, Elsevier Inc., Oxford, UK, 2008.

[16] W. Layton, Weak imposition of "no-slip" conditions in finite element methods., Computers and Mathematics with Applications 38 (1999) 129-142.

[17] J.-L. Barrat, L. Bocquet, Influence of wetting properties on hydrodynamic boundary conditions at a fluid/solid interface, Faraday Discuss 112 (1999) $119-128$.

[18] R. Pit, H. Hervet, L. Lger, Friction and slip of a simple liquid at a solid surface, Tribology Letters 7 (2-3) (1999) 147-152.

[19] Y. Zhu, S. Granick, Limits of the hydrodynamic no-slip boundary condition, Physical Review Letters 88 (2002) 106102.

[20] L. M. Hocking, A moving fluid interface on a rough surface, Journal of Fluid Mechanics 76 (04) (1976) 801-817. 
[21] J. De Hart, G. W. M. Peters, P. J. G. Schreurs, F. P. T. Baaijens, A twodimensional fluid-structure interaction model of the aortic value, Journal of Biomechanics 33 (9) (2000) 1079-1088.

[22] D. Goldstein, R. Handler, L. Sirovich, Modeling a no-slip flow boundary with an external force field, Journal of Computational Physics 105 (2) (1993) 354-366.

[23] R. van Loon, P. Anderson, F. van de Vosse, A fluid-structure interaction method with solid-rigid contact for heart valve dynamics, Journal of Computational Physics 217 (2) (2006) 806-823.

[24] C. B. Millikan, On the steady motion of viscous, incompressible fluids; with particular reference to a variation principle, Philosophical Magazine Series 77 (44) (1929) 641-662.

[25] C. Lanczos, The Variational Principles of Mechanics, 4th Edition, Courier Dover Publications, 1970. 

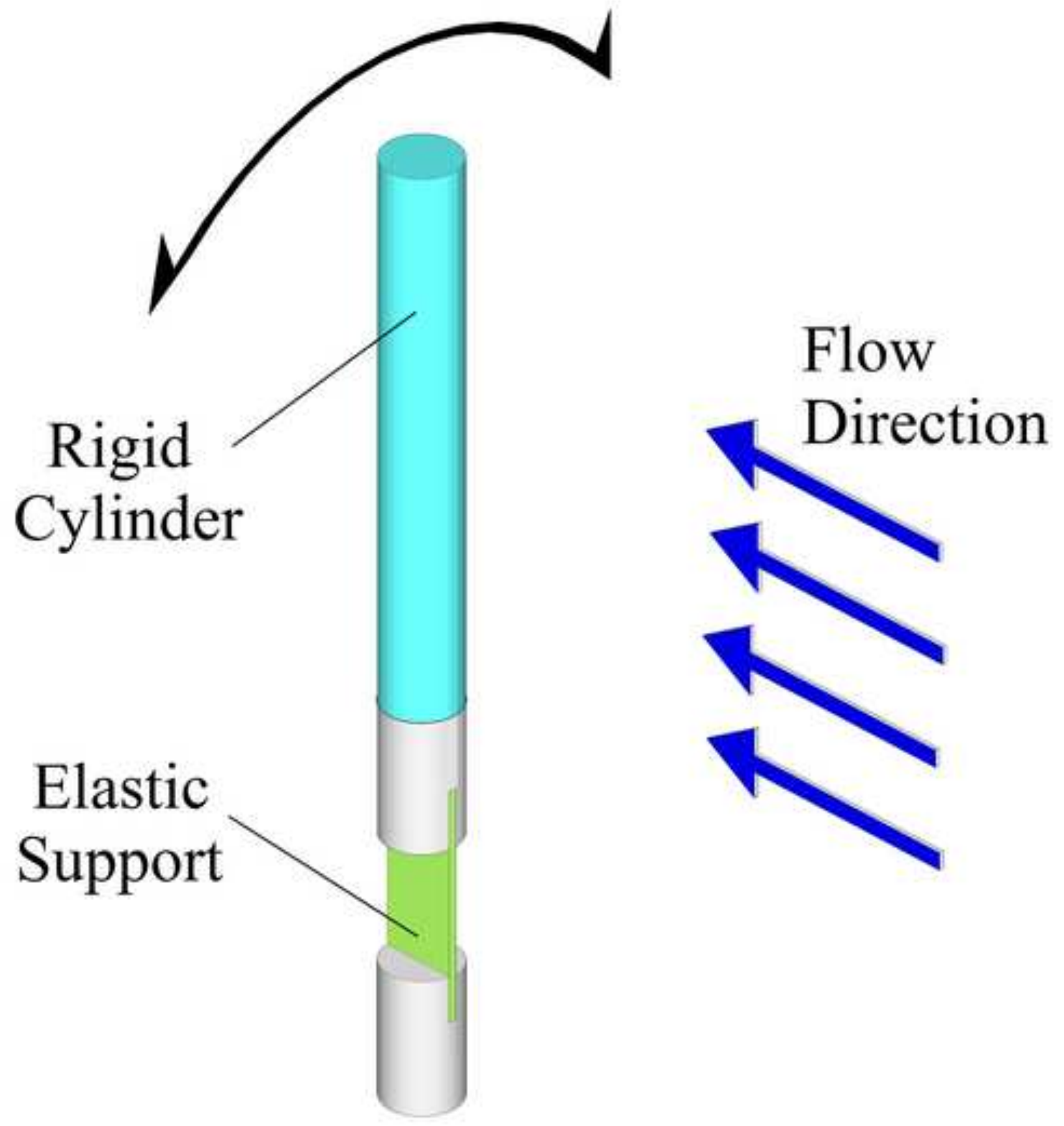

Flow Direction
Elastic Support
Figure 1b 


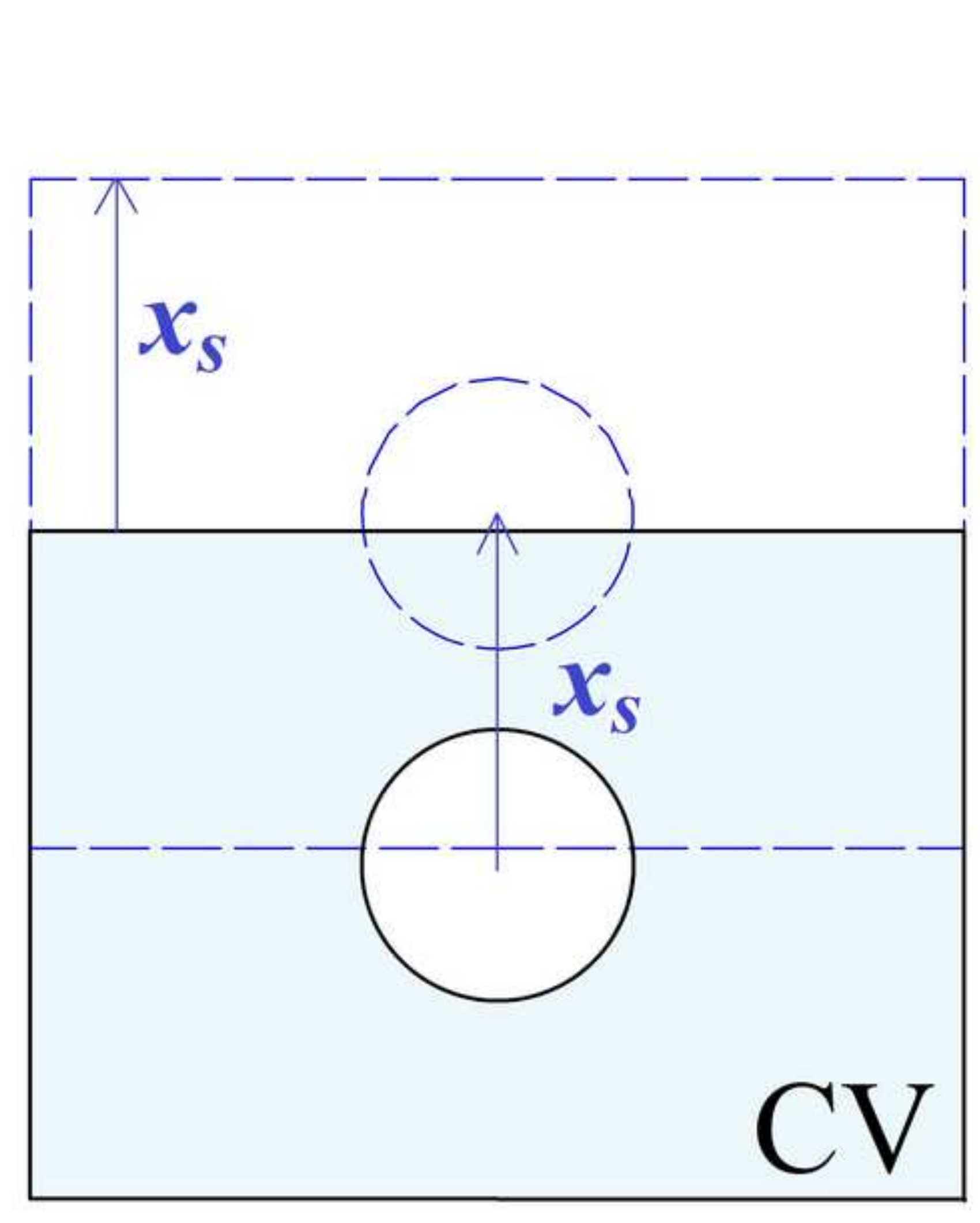

Figure 3

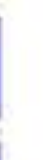

3

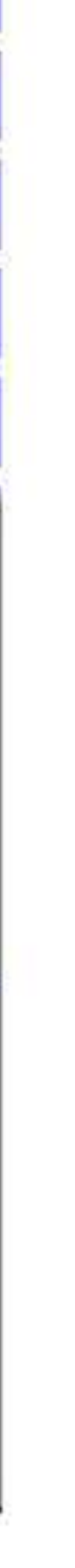


Figure 4

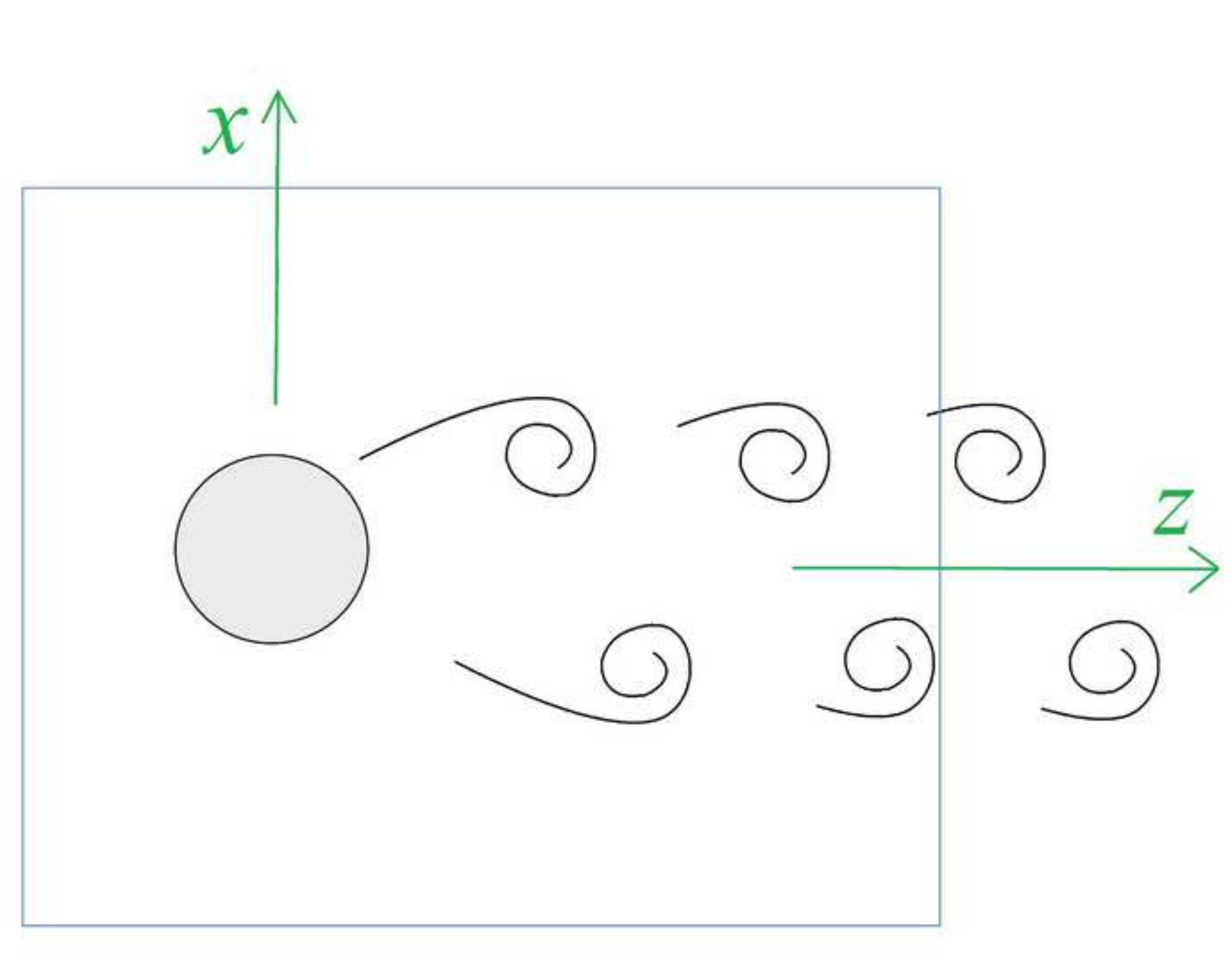

